\title{
WELL-POSEDNESS OF A MATHEMATICAL MODEL FOR ALZHEIMER'S DISEASE
}

\author{
MICHIEL BERTSCH, BRUNO FRANCHI, MARIA CARLA TESI, AND ANDREA TOSIN
}

\begin{abstract}
We consider the existence and uniqueness of solutions of an initial-boundary value problem for a coupled system of PDE's arising in a model for Alzheimer's disease. Apart from reaction diffusion equations, the system contains a transport equation in a bounded interval for a probability measure which is related to the malfunctioning of neurons. The main ingredients to prove existence are: the method of characteristics for the transport equation, a priori estimates for solutions of the reaction diffusion equations, a variant of the classical contraction theorem, and the Wasserstein metric for the part concerning the probability measure. We stress that all hypotheses on the data are not suggested by mathematical artefacts, but are naturally imposed by modelling considerations. In particular the use of a probability measure is natural from a modelling point of view. The nontrivial part of the analysis is the suitable combination of the various mathematical tools, which is not quite routine and requires various technical adjustments.
\end{abstract}

\section{INTRODUCTION}

In [3, 4] a macroscopic mathematical model was proposed which describes the onset and evolution of Alzheimer's disease (AD). This model is meant to mirror the so-called Amyloid Cascade Hypothesis [1, 15, 21], coupled with the spreading of the disease through neuron-to-neuron transmission (prionoid hypothesis [5, 23]). Alzheimer's disease (AD) is the prevalent form of late life dementia. Its global prevalence, about 24 millions in 2011, is expected to double in 20 years [19].

In order to clarify the structure of our equations and the choice of our assumptions, let us sketch a gist of their biological background. We refer to [3, 4, for a complete description of the model and an account of the most recent biomedical literature. The model focusses on the role of the polymer beta-amyloid, in particular its toxic soluble isoform $\mathrm{A} \beta_{42}$. Monomers of $\mathrm{A} \beta_{42}$ are regularly produced by neurons and successively cleared - among others - by the microglia. In the last decades, researchers have observed that an imbalance between production and clearance of $\mathrm{A} \beta_{42}$ (shortly $\mathrm{A} \beta$ from now on) is a very early, often initiating factor in $\mathrm{AD}$. Soluble $\mathrm{A} \beta$ diffuses through the microscopic tortuosity of the brain tissue and undergoes an agglomeration process. Eventually this leads to the formation of long, insoluble fibrils, accumulating in spherical deposits known as senile plaques that are solid deposits of even larger aggregates of $\mathrm{A} \beta$; nowadays, plaques are not considered neurotoxic, but are usually abundantly present in the brain of ADpatients (though they can be present in old brains without any symptom of dementia). Plaques are clinically observable through medical imaging using a special type of PET (Positron Emission Tomography) scan.

Below we briefly describe the model. The main purpose of the present paper is to establish its mathematical well-posedness. Mathematically, our model consists of a transport equation coupled with a system of nonlinear diffusion equations (a Smoluchowski-type system with diffusion). Due to the very nature of the biological phenomena we are studying, the main feature of such a system is that the transport velocity depends on the solution of the Smoluchowski equation, which, in turn, contains a source term that depends on the solution of the transport equation, so that the two groups of equations cannot be uncoupled. For an introduction to the use of transport equations in mathematical models of life sciences, we refer the reader to [18].

Key words and phrases. Transport and diffusion equations; Smoluchowski equations; mathematical models of Alzheimer's disease. 
Let us give a less cursory description of the system which we consider, from both a mathematical and biomedical point of view. We do not enter the biological details and merely mention those which are related to the structure of our equations. We would like to stress that the equations of our model involve only functions that have a precise qualitative clinical counterpart in routinely observable phenomena: the health state of the different brain regions (by means of a PET measuring the cerebral glucose metabolism), the amount of $\mathrm{A} \beta$ in the cerebral spinal fluid, and the $\mathrm{A} \beta$ plaques (by means of amyloid-PET scans).

Let $\Omega \subseteq \mathbb{R}^{n}$ be a portion of cerebral tissue. The molar concentration of soluble $\mathrm{A} \beta$ polymers of length $m$ at $x \in \Omega$ and time $t \geq 0$ is denoted by $u_{m}(x, t)(1 \leq m<N)$, that of clusters of oligomers of length greater or equal to $N$ (the plaques) by $u_{N}(x, t)$. We use a parameter $a$, ranging from 0 to 1 , to describe the degree of malfunctioning of a neuron; a close to 0 stands for "the neuron is healthy" and $a$ close to 1 for "the neuron is dead". Given $x \in \Omega$ and $t \geq 0, f=f_{x, t}$ is a probability measure and $d f_{x, t}(a)$ denotes the fraction of neurons at $x$ and time $t$ with degree of malfunctioning between $a$ and $a+d a$. The progression of $\mathrm{AD}$ is mainly determined by the deterioration rate of the health state of the neurons, $v=v_{x}(a, t) \geq 0$. We use the notation $v[f]$ to stress its dependence on $f$ :

$$
(v[f])_{x}(a, t):=\int_{\Omega}\left(\int_{[0,1]} \mathcal{K}(x, a, y, b) d f_{y, t}(b)\right) d y+\mathcal{S}\left(x, a, u_{1}(x, t), \ldots, u_{N-1}(x, t)\right) .
$$

The term $\mathcal{S} \geq 0$ in (11) models the action of toxic A $\beta$ oligomers. For example, assuming that the toxicity of soluble $\mathrm{A} \beta$-polymers is proportional to their total mass and introducing a threshold value $\bar{U}>0$ for the amount of toxic $\mathrm{A} \beta$ needed to damage neurons, a possible choice for $\mathcal{S}$ is

$$
\mathcal{S}=C_{\mathcal{S}}(1-a)\left(\sum_{m=1}^{N-1} m u_{m}(x, t)-\bar{U}\right)^{+}, \quad \text { where } p^{+}:=\max \{p, 0\}
$$

see also 3 for a more detailed discussion.

The integral term in (1) describes the possible propagation of AD through the neural pathway. Malfunctioning neighbours are harmful for a neuron's health state, while healthy ones are not: $\mathcal{K}(x, a, y, b) \geq 0$ for all $x, y \in \Omega$ and $a, b \in[0,1]$ and

$$
\mathcal{K}(x, a, y, b)=0 \quad \text { if } a>b .
$$

For the sake of simplicity we choose $\mathcal{K}(x, a, y, b)=\mathcal{G}_{x}(a, b) h(|x-y|)$, where $h(r)$ is a nonnegative and decreasing function which vanishes at some $r=r_{0}$ and satisfies $\int_{|y|<r_{0}} h(|y|) d y=1$. For instance, in [3] the following form of $\mathcal{G}_{x}$ is used: $\mathcal{G}_{x}(a, b)=C_{\mathcal{G}}(b-a)^{+}$, which does not depend explicitly on $x$. In the limit $r_{0} \rightarrow 0$, (11) reduces to

$$
(v[f])_{x}(a, t)=\int_{[0,1]} \mathcal{G}_{x}(a, b) d f_{x, t}(b)+\mathcal{S}\left(x, a, u_{1}(x, t), \ldots, u_{N-1}(x, t)\right) .
$$

We shall henceforth use (3) for the deterioration rate $v[f]$.

In view of the meaning of the rate $v$, the equation for $f$ is given by

$$
\partial_{t} f+\partial_{a}(f v[f])=J[f] .
$$

The term $J[f]$ represents the onset of AD: we assume that in small (randomly chosen) parts of the cerebral tissue, concentrated for instance in the hippocampus and described by a characteristic function $\chi(x, t)$, the degree of malfunctioning of neurons randomly jumps to higher values due to external agents or genetic factors. More precisely, $(J[f])_{x, t}$ denotes the measure

$$
d(J[f])_{x, t}(a):=\eta(t) \chi(x, t)\left[\left(\int_{[0,1]} P(t, b, a) d f_{x, t}(b)\right) d a-d f_{x, t}(a)\right]
$$


where the function $P(t, b, a)$ is the probability to jump from state $b$ to state $a$ (which vanishes if $a<b)$ and $\eta>0$ is the jump frequency. A possible choice is

$$
P(t, b, a) \equiv P(b, a)= \begin{cases}\frac{2}{1-b} & \text { if } b \leq a \leq \frac{1}{2}(1+b) \\ 0 & \text { otherwise }\end{cases}
$$

It is worth stressing that the choice of looking for a measure $f_{x, t}$ comes from the model itself. In fact, a "healthy brain" would correspond to $f_{x, t}(a)=\delta(a)$, where $\delta$ is the Dirac measure centred at the origin.

Now we are ready to write the system of equations for $f, u_{1}, \cdots, u_{N}$ :

$$
\begin{cases}\partial_{t} f+\partial_{a}(f v[f])=J[f] & \text { in } \Omega \times[0,1] \times(0, T] \\ \varepsilon \partial_{t} u_{1}-d_{1} \Delta u_{1}=R_{1}:=-u_{1} \sum_{j=1}^{N} a_{1, j} u_{j}+\mathcal{F}[f]-\sigma_{1} u_{1} & \text { in } Q_{T}=\Omega \times(0, T] \\ \varepsilon \partial_{t} u_{m}-d_{m} \Delta u_{m}=R_{m}:=\frac{1}{2} \sum_{j=1}^{m-1} a_{j, m-j} u_{j} u_{m-j} & \\ \quad-u_{m} \sum_{j=1}^{N} a_{m, j} u_{j}-\sigma_{m} u_{m} & \text { in } Q_{T} \quad(2 \leq m<N) \\ \varepsilon \partial_{t} u_{N}=\frac{1}{2} \sum_{\substack{j+k \geq N \\ k, j<N}} a_{j, k} u_{j} u_{k} & \text { in } Q_{T} .\end{cases}
$$

Here $\varepsilon>0$ is a small parameter which expresses the existence of two time scales: processes which determine the dynamics of $\mathrm{A} \beta$ (production, aggregation, diffusion, deposition) occur on a much smaller time scale (hours) than the evolution of the disease (years). The diffusion coefficients $d_{m}$ depend on the length of the polymer (longer polymers diffuse less); plaques do not diffuse. The quadratic terms (in $u_{i}$ ) model the aggregation of $\mathrm{A} \beta$ polymers, according to the Smoluchowski equations. We refer to 1, 10 for an extensive discussion of the aggregation mechanism and the choice of the coagulation rates $a_{i, j}$. The linear terms $-\sigma_{m} u_{m}$ model the phagocytic activity of the microglia and other bulk clearance processes [14].

We stress that system (6) is fully coupled, because the transport equation for $f$ contains a dependence on $u_{1}, \ldots, u_{N-1}$ in the deterioration rate $v[f]$, cf. (3). Notice that if $\mathcal{S} \equiv 0$ in (3) then the equation for $f$ decouples from the rest of the system and may be possibly studied alone by relying on the results reported in [6, 7]. Nevertheless, the assumption $\mathcal{S} \equiv 0$ is not a minor issue in the modelling of AD spreading, because it would imply a propagation of the disease due only to prionic diffusion, which is a controversial topic in the medical literature. For this reason, in our model we prefer to take into account also the toxic contribution of A $\beta$ oligomers, i.e. $\mathcal{S} \neq 0$ in (3), which requires to study system (6) as a whole.

$\mathrm{A} \beta$ monomers are produced by neurons. Their production increases if neurons are damaged, and a possible choice for the source term $\mathcal{F}$ in the equation for $u_{1}$ is

$$
\mathcal{F}[f](x, t)=C_{\mathcal{F}} \int_{0}^{1}\left(\mu_{0}+a\right)(1-a) d f_{x, t}(a) .
$$

The small constant $\mu_{0}>0$ accounts for $\mathrm{A} \beta$ production by healthy neurons.

We assume that $\partial \Omega$ consists of smooth disjunct boundaries, $\partial \Omega_{0}$ and $\partial \Omega_{1}$, where $\partial \Omega_{1}$ represents the disjunct union of the boundaries of the cerebral ventricles through which $\mathrm{A} \beta$ is removed from the cerebrospinal fluid (CSF) by an outward flow through the choroid plexus (cf. [14, 22]). In the present paper we solve system ([6) with appropriate initial-boundary conditions:

$$
\begin{cases}f_{x, 0}=\left(f_{0}\right)_{x} & \text { if } x \in \Omega \\ u_{i}(x, 0)=u_{0 i}(x) & \text { if } x \in \Omega, 1 \leq i \leq N \\ \partial_{n} u_{i}(x, t)=0 & \text { if } x \in \partial \Omega_{0}, t>0,1 \leq i<N \\ \partial_{n} u_{i}(x, t)=-\gamma_{i} u_{i}(x, t) & \text { if } x \in \partial \Omega_{1}, t>0,1 \leq i<N\end{cases}
$$

where $n$ is the outward pointing normal on $\partial \Omega$. 
In Section 2 we describe the hypotheses on the data and formulate the main result on global well-posedness. In Section 3 we rewrite the system in terms of the characteristics of the transport equation for $f$ and show that the new system is equivalent to the original one. We point out that under our assumptions the characteristics exist in the classical sense. The major difficulty arises from the strong nonlinearity of the system: the transport equation depends nonlinearly on both its solution (through an integral operator) and the solution of the Smoluchowski system, which in turn depends on the solution of the transport equation. In Section 4 we use a contraction argument to prove local existence and uniqueness; not surprisingly, the metric for the probability measures $f$ will involve Wasserstein distances. The fact that the Wasserstein distance $\mathcal{W}_{1}$ depends on the action of the measures on Lipschitz functions yields a technical difficulty when we try to apply an iteration argument in order to obtain the local existence of a solution. This difficulty can be bypassed thanks to an ad hoc formulation of the standard fixed point theorem. Finally, in Section 5 we prove a priori bounds which imply global existence. In Appendix A we collect some technical facts about probability measures and Wasserstein distances to make this paper as self-consistent as possible.

We conclude with some comments.

For more details on the model we refer to [3, which also contains some two-dimensional simulations and qualitative comparison with experimental data. A derivation of the transport equation for $f$ from a Boltzmann-type kinetic approach is contained in [4].

The macroscopic model which we have briefly described, and in particular the use of the Smoluchowski equations to model the aggregation of $\mathrm{A} \beta$, is based on an earlier microscopical model described in [1, 10]. The passage from that microscopic aggregation-diffusion model to Smoluchowski equations with a source term as in (6) is obtained by a two-scale homogenization technique in [8, 9].

For the moment the model deliberately neglects some important aspects of the disease such as the role of the tau-protein, but in a future paper we shall extend the model and make it more realistic. Nevertheless, the term $\mathcal{G}$ in the deterioration rate for the equation of $f$ can already be thought of as taking into account a "prionic" model of the spread of the disease, and associated with the diffusion of the intraneural tau-protein (see, e,g., [13, 23]). Despite the extreme complexity of $\mathrm{AD}$, mathematically such an extension is doable due to the high degree of flexibility of the modelling approach. The major difficulty is the lack of both experimental data and detailed knowledge about the relevant biomedical processes, but fortunately biomedical research on AD evolves rapidly.

In this context we also mention a recent paper by Hao and Friedman [12, which does take into account a higher degree of AD's complexity and contains simulations of medical therapies; the authors however do not consider AD's initial stage. A major challenge is how to diagnose AD's early stage and develop therapies to slow down its further development.

\section{Problem Statement And MAin RESUlts}

Throughout the paper we set $T>0, N \in \mathbb{N}$, while $\Omega \subset \mathbb{R}^{n}$ is an open and bounded set with a smooth boundary $\partial \Omega$, which is the disjunct union of $\partial \Omega_{0}$ and $\partial \Omega_{1}$.

To treat the measures $f_{x, t}$ we introduce a metric space $X_{[0,1]}$ :

Definition 2.1. The space $\mathcal{P}([0,1])$ of probability measures on $[0,1]$ endowed with the Wasserstein distance $\mathcal{W}_{1}$ is denoted by $X_{[0,1]}$.

We refer to [2] for the definition of the Wasserstein distances $\mathcal{W}_{p}$. By Proposition A.3. $X_{[0,1]}$ is a complete separable metric space. By Proposition A.7, a sequence $\left(\mu_{n}\right)_{n \in \mathbb{N}}$ converges in $X_{[0,1]}$ if and only if it converges narrowly or weakly*.

We denote by $C\left([0, T] ; X_{[0,1]}\right)$ the space of continuous functions from the interval $[0, T]$ to $X_{[0,1]}$. Endowed with the distance

$$
\max _{0 \leq t \leq T} \mathcal{W}_{1}\left(\left(\mu_{1}\right)_{t},\left(\mu_{2}\right)_{t}\right)
$$

also $C\left([0, T] ; X_{[0,1]}\right)$ is a complete metric space. 
2.1. Hypotheses on the data. Throughout the paper we shall use the following assumptions on the data (below $\partial_{a}, \nabla_{u}$ etc. denote distributional derivatives; $C$ denotes a generic constant):

$\left(H_{1}\right) \varepsilon, C_{\mathcal{F}}, \mu_{0}, d_{i}, \sigma_{i}, \gamma_{i}, a_{i, j}$ are positive constants $(1 \leq i<N, 1 \leq j \leq N)$;

$\left(H_{2}\right) \quad u_{0 i} \in C(\bar{\Omega})$ is nonnegative $(i=1, \cdots, N)$, and $\left(\bar{f}_{0}\right)_{x} \in X_{[0,1]}$ for a.e. $x \in \Omega$;

$\left(H_{3}\right) \chi$ is the characteristic function of a measurable set $Q_{0} \subseteq Q_{T}=\Omega \times[0, T]$; the function $\eta \in C([0, T])$ is nonnegative;

$\left(H_{4}\right)$ for a.e. $x \in \Omega, \mathcal{G}_{x} \in C\left([0,1]^{2}\right), \mathcal{G}_{x}(1, b)=0$ for $b \in[0,1]$, and

$$
-C \leq \partial_{a} \mathcal{G}_{x} \leq 0,\left|\partial_{b} \mathcal{G}_{x}\right| \leq C \quad \text { in }[0,1]^{2} \text {; }
$$

$\left(H_{5}\right) \mathcal{S} \in L^{\infty}\left(\Omega ; C\left([0,1] \times[0, \infty)^{N-1}\right)\right), \mathcal{S}\left(x, 1, u_{1}, \ldots, u_{N-1}\right)=0$ for $u_{i} \geq 0$ and a.e. $x \in \Omega$, and for all compact sets $K \subset[0, \infty)^{N-1}$ there exists a constant $C(K)$ such that for a.e. $x \in \Omega$

$$
-C(K) \leq \partial_{a} \mathcal{S}(x, a, u) \leq 0,\left|\nabla_{u} \mathcal{S}(x, a, u)\right| \leq C(K) \quad \text { for } a \in[0,1], u \in K
$$

$\left(H_{6}\right) \quad P \in C\left([0, T] \times[0,1]^{2}\right), P$ is nonnegative, for all $t \in[0, T]$

$$
\int_{0}^{1} P(t, b, a) d a=1 \quad \text { for } b \in[0,1], \quad P(t, b, a)=0 \quad \text { if } a<b
$$

and there exists $L>0$ such that for all $a^{\prime}, a^{\prime \prime}, b^{\prime}, b^{\prime \prime} \in[0,1]$ and $t \in[0, T]$

$$
\left|P\left(t, b^{\prime}, a^{\prime}\right)-P\left(t, b^{\prime \prime}, a^{\prime \prime}\right)\right| \leq L\left(\left|b^{\prime}-b^{\prime \prime}\right|+\left|a^{\prime}-a^{\prime \prime}\right|\right) .
$$

2.2. Main result. We introduce some additional notation. Let $\mathcal{M}(0,1)$ be the space of signed Radon measures on the interval $(0,1)$. Then $\mathcal{M}(0,1)$ is the dual of $C([0,1])$, and $\mu: \Omega \times(0, T) \rightarrow$ $\mathcal{M}(0,1)$ is said to be weakly* measurable if for any $\rho \in C([0,1])$ the map

$$
(x, t) \mapsto \int \rho(a) d \mu_{x, t}(a)
$$

is measurable in $\Omega \times(0, T)$. We say that

$$
f \in \mathcal{L}\left(\Omega ; C\left([0, T] ; X_{[0,1]}\right)\right)
$$

if $\left.f \in C\left([0, T] ; X_{[0,1]}\right)\right)$ for a.e. $x \in \Omega$ and $f$ is weakly* measurable as a function from $\Omega \times(0, T)$ in $\mathcal{M}(0,1)$. In particular, if $f \in \mathcal{L}\left(\Omega ; C\left([0, T] ; X_{[0,1]}\right)\right)$, then, by the Fubini-Tonelli Theorem, for all $\psi \in C([0,1] \times \bar{\Omega} \times[0, T])$

$$
x \mapsto \int_{0}^{T}\left(\int \psi(a, x, t) d f_{x, t}(a)\right) d t \quad \text { belongs to } L^{\infty}(\Omega) .
$$

Definition 2.2. A $(N+1)$-ple $\left(f, u_{1}, \cdots, u_{N}\right)$ is called a solution of problem (6)-(8) in $[0, T]$ if

(i) $f \in \mathcal{L}\left(\Omega ; C\left([0, T] ; X_{[0,1]}\right)\right)$;

(ii) $u_{i} \in C\left(\bar{Q}_{T}\right)$ and $u_{i} \geq 0$ in $Q_{T}$ for $1 \leq i \leq N$;

(iii) the first equation in ([6]) is satisfied in a weak sense: for a.e. $x \in \Omega$

$$
\int_{0}^{\tau}\left(\int\left(\partial_{t} \phi+v_{x} \partial_{a} \phi\right) d f_{x, t}+\int \phi d J_{x, t}\right) d t=\int \phi(\cdot, \tau) d f_{x, \tau}-\int \phi(\cdot, 0) d\left(f_{0}\right)_{x}
$$

for all $\tau \in[0, T]$ and $\phi \in C^{1}([0,1] \times[0, T])$, where the function $v$ is defined by (3) and the signed measure $J$ by (15);

(iv) if $1 \leq i<N, u_{i} \in L^{2}\left([0, T] ; H^{1}(\Omega)\right)$ and

$$
\begin{gathered}
d_{i} \int_{0}^{T}\left[\int_{\Omega} \nabla u_{i}(x, s) \cdot \nabla \psi(x, s) d x+\gamma_{i} \int_{\partial \Omega_{1}} u_{i}(x, s) \psi(x, s) d \sigma(x)\right] d s \\
=\varepsilon \iint_{Q_{T}} u_{i} \psi_{t}+\varepsilon \int_{\Omega} u_{0 i} \psi(x, 0) d x+\iint_{Q_{T}} R_{i} \psi
\end{gathered}
$$

for all $\psi \in H^{1}\left([0, \tau] ; H^{1}(\Omega)\right), \psi(x, \tau)=0$, where $R_{i}$ is defined as in (6) and $\mathcal{F}$ (which is part of $\left.R_{1}\right)$ by (7);

(v) $\partial_{t} u_{N} \in C\left(\bar{Q}_{T}\right), u_{N}(\cdot, 0)=u_{0 N}$ in $\Omega$, and the equation for $u_{N}$ in (6) is satisfied in $Q_{T}$. 
Remark 2.3. (a) It follows from (5) and $\left(H_{6}\right)$ that, for a.e. $x \in \Omega, \int d J_{x, t}=0$ for $t \in[0, T]$. (b) The concept of weak solution of the first order transport equation, defined in Definition 2.2 ( $(i i i)$, needs some explanation. It follows from (9)-(10) that, for a.e. $x \in \Omega, v$ is uniformly Lipschitz continuous with respect to $a$ :

$$
\partial_{a} v_{x}(a, t)=\int_{[0,1]} \partial_{a} \mathcal{G}(x, a, b) d f_{x, t}(b)+\partial_{a} \mathcal{S}\left(x, a, u_{1}(x, t), \ldots, u_{N-1}(x, t)\right) \leq 0 .
$$

In particular it follows from $\left(H_{4-5}\right)$ that, for a.e. $x \in \Omega, v_{x}(1, t)=0$ for $t \in[0, T]$ and $v_{x}(a, t) \geq 0$ for $a \in[0,1]$ and $t \in[0, T]$. This implies that formally the "flux" $f v$ vanishes at $a=1$, a condition which is made precise by the choice of continuous test functions $\phi(x, a, t)$ without any restriction at $a=1$. Since $v \geq 0$ at $a=0$, characteristics (see the next section) "enter the domain $[0,1]$ " at $a=0$; so we need a boundary condition at $a=0$ which, according to Definition 2.2 ( $i i i)$, is again the no-flux condition. Actually this is imposed by the condition that $f_{x, \tau}$ is a probability measure in $[0,1]$ : choosing $\phi \equiv 1$ it follows from Definition 2.2 (iii) and Remark 2.3(a) that for a.e. $x \in \Omega$

$$
\int d f_{x, \tau}=\int d\left(f_{0}\right)_{x}=1 \quad \text { for } \tau \in(0, T]
$$

The main result of the paper is the following well-posedness result.

Theorem 2.4. Let $\Omega \subset \mathbb{R}^{n}$ be an open and bounded set with a smooth boundary $\partial \Omega$, which is the disjunct union of $\partial \Omega_{0}$ and $\partial \Omega_{1}$. Let $T>0$ and $N \in \mathbb{N}$, and let hypotheses $\left(H_{1-6}\right)$ be satisfied. Then problem (6) -(8) has a unique solution in $[0, T]$ in the sense of Definition [2.2.

\section{The CHARACTERISTICS}

Let $f \in \mathcal{L}\left(\Omega ; C\left([0, T] ; X_{[0,1]}\right)\right)$ and $u_{i} \in C\left(\bar{Q}_{T}\right)$, and let $v[f]$ be defined by (3). By the Lipschitz continuity of $a \mapsto v_{x}(a, t)$ (see Remark 2.3(b)), for a.e. $x \in \Omega$ the problem for the characteristic issued from $y \in[0,1]$,

$$
\left\{\begin{array}{l}
\partial_{t} A_{x}(y, t)=v_{x}\left(A_{x}(y, t), t\right) \quad \text { for } 0<t \leq T \\
A_{x}(y, 0)=y
\end{array}\right.
$$

has a unique solution which satisfies

$$
\begin{cases}0 \leq A_{x}\left(y_{1}, t\right)<A_{x}\left(y_{2}, t\right) \leq A_{x}(1, t)=1 & \text { if } 0 \leq y_{1}<y_{2} \leq 1,0 \leq t \leq T \\ A_{x}\left(y, t_{1}\right) \leq A_{x}\left(y, t_{2}\right) & \text { if } y \in[0,1], 0 \leq t_{1} \leq t_{2} \leq T\end{cases}
$$

Observe that, for a.e. $x \in \Omega$, the function $y \mapsto A_{x}(y, t)$ is continuous and

$$
\partial_{y} A_{x}(y, t)=\exp \left(\int_{0}^{t} \partial_{a} v_{x}\left(A_{x}(y, s), s\right) d s\right)>0 \quad \text { for all } t \in[0, T] .
$$

In particular for a.e. $x \in \Omega$ the function $y \mapsto A_{x}(y, t)$ is injective for all $t \in[0, T]$.

Below we shall reformulate problem (6)-(8) in terms of the characteristics, but before doing so we prove the following result.

Proposition 3.1. Let $f \in \mathcal{L}\left(\Omega ; C\left([0, T] ; X_{[0,1]}\right)\right)$ and $u_{i} \in C\left(\bar{Q}_{T}\right)$. Let $v[f]$ and $J[f]$ be defined by (3) and (5). Let, for a.e. $x \in \Omega, A_{x}(y, t)$ be the solution of (16) for any $y \in[0,1]$. If $f$ satisfies (41) in the sense of Definition [2.2(iii), then, for a.e. $x \in \Omega$,

$$
\operatorname{supp} f_{x, t}, \operatorname{supp} J_{x, t} \subseteq\left[A_{x}(0, t), 1\right] \quad \text { for } t \in(0, T] .
$$

Proof. $A_{x}$ is well defined for a.e. $x \in \Omega$. We fix such $x$ and also $\tau \in(0, T]$. Let $h \in C^{1}(\mathbb{R})$ be nondecreasing and satisfy $h \equiv 0$ in $(-\infty, 0]$ and $h \equiv 1$ in $[1, \infty)$. Let $\delta>0$ and set for a.e. $x \in \Omega$

$$
h_{\delta}(s)=h(s / \delta) \text { for } s \in \mathbb{R} ; \quad \psi_{\delta}(a, t)=h_{\delta}\left(A_{x}(0, t)-a\right) \text { for } a \in[0,1], t \in[0, T] .
$$

Then $\psi_{\delta}$ is of class $C^{1}$ and

$$
\partial_{a} \psi_{\delta}=-\frac{1}{\delta} h^{\prime}\left(\frac{A_{x}(0, t)-a}{\delta}\right), \quad \partial_{t} \psi_{\delta}=\frac{1}{\delta} v_{x}\left(A_{x}(0, t), t\right) h^{\prime}\left(\frac{A_{x}(0, t)-a}{\delta}\right) .
$$


We use $\psi_{\delta}$ as a test function in Definition $2.2($ iii $)$. Since $A_{x}(0,0)=0, \psi_{\delta}(a, 0)=0$ if $a \geq 0$ and $\int \psi_{\delta}(\cdot, 0) d\left(f_{0}\right)_{x}=0$. Therefore the test function relation implies that

$$
\int \psi_{\delta}(\cdot, \tau) d f_{x, \tau}-\int_{0}^{\tau}\left(\int \psi_{\delta} d J_{x, t}\right) d t \rightarrow 0 \quad \text { as } \delta \rightarrow 0
$$

if we prove that

$$
\int_{0}^{\tau}\left(\int\left(\partial_{t} \psi_{\delta}+v_{x} \partial_{a} \psi_{\delta}\right) d f_{x, t}\right) d t \rightarrow 0 \quad \text { as } \delta \rightarrow 0
$$

To prove (21) we observe that

$$
\left|\partial_{t} \psi_{\delta}+v_{x} \partial_{a} \psi_{\delta}\right|=\left|\frac{v_{x}\left(A_{x}(0, t), t\right)-v_{x}(a, t)}{\delta} h^{\prime}\left(\frac{A_{x}(0, t)-a}{\delta}\right)\right| \leq C \sup _{s \in \mathbb{R}}\left|\operatorname{sh}^{\prime}(s)\right|
$$

for some constant $C$ which does not depend on $\delta$, whence

$$
\begin{aligned}
& \left|\int_{0}^{\tau}\left(\int\left(\partial_{t} \psi_{\delta}+v_{x} \partial_{a} \psi_{\delta}\right) d f_{x, t}\right) d t\right| \\
& \quad \leq C \int_{0}^{\tau}\left(\int d f_{x, t}\left\llcorner\left(A_{x}(0, t)-\delta, A_{x}(0, t)\right) \cap\left[0, A_{x}(0, t)\right)\right) d t .\right.
\end{aligned}
$$

Here and in the following, the symbol $L$ denotes the restriction of a measure to a measurable subset, see [16, Definition 1.8]. Since $\bigcap_{\delta>0}\left(A_{x}(0, t)-\delta, A_{x}(0, t)\right) \cap\left[0, A_{x}(0, t)\right)=\emptyset$ and

$$
\mid \int d f_{x, t}\left\llcorner\left(A_{x}(0, t)-\delta, A_{x}(0, t)\right) \cap\left[0, A_{x}(0, t)\right) \mid \leq 1 \quad \text { for } t \in[0, \tau],\right.
$$

(21) follows from (22) and the Dominated Convergence Theorem.

By (20) and the Dominated Convergence Theorem,

$$
\int d f_{x, \tau}\left\llcorner\left[0, A_{x}(0, \tau)\right)=\int_{0}^{\tau}\left(\int d J_{x, t}\left\llcorner\left[0, A_{x}(0, t)\right)\right) d t .\right.\right.
$$

It follows from (5), the Fubini-Tonelli Theorem and (11) that

$$
\begin{aligned}
\int & d J_{x, t}(a)\left\llcorner\left[0, A_{x}(0, t)\right)\right. \\
= & \eta(t) \chi(x, t)\left[\int\left(\int_{0}^{A_{x}(0, t)} P(t, b, a) d a\right) d f_{x, t}(b)-\int d f_{x, t}(a)\left\llcorner\left[0, A_{x}(0, t)\right)\right]\right. \\
= & \eta \chi\left[\int ( \int _ { b } ^ { A _ { x } ( 0 , t ) } P ( t , b , a ) d a ) d f _ { x , t } ( b ) \left\llcorner\left[0, A_{x}(0, t)\right)-\int d f_{x, t}(a)\left\llcorner\left[0, A_{x}(0, t)\right)\right]\right.\right. \\
\leq \eta \chi & \int d f_{x, t}(b)\left\llcorner\left[0, A_{x}(0, t)\right)-\int d f_{x, t}(a)\left\llcorner\left[0, A_{x}(0, t)\right)\right]=0 .\right.
\end{aligned}
$$

Combined with (23), this implies (19).

We now reformulate the original problem in terms of the characteristics. Specifically, we shall see below that the measure $f$ can be obtained by transporting along the characteristics a suitable 
measure $g$ (i.e., $f$ is the push forward of $g$ through $A$, cf. Definition A.1), which satisfies:

$$
\left\{\begin{array}{l}
\partial_{t} A_{x}(y, t)=\int \mathcal{G}_{x}\left(A_{x}(y, t), A_{x}(\xi, t)\right) d g_{x, t}(\xi)+\mathcal{S}\left(x, A_{x}(y, t), u_{1}, \ldots, u_{N-1}\right) \\
\partial_{t} g_{x, t}(y)=\eta \chi\left[\partial_{y} A_{x}(y, t) \int P\left(t, A_{x}(\xi, t), A_{x}(y, t)\right) d g_{x, t}(\xi)-g_{x, t}(y)\right] \\
\varepsilon \partial_{t} u_{1}-d_{1} \Delta u_{1}=\tilde{R}_{1}:=-u_{1} \sum_{j=1}^{N} a_{1, j} u_{j}-\sigma_{1} u_{1}+C_{\mathcal{F}} \int\left(\mu_{0}+A_{x}(\xi, t)\right)\left(1-A_{x}(\xi, t)\right) d g_{x, t}(\xi) \\
\varepsilon \partial_{t} u_{m}-d_{m} \Delta u_{m}=\tilde{R}_{m}:=\frac{1}{2} \sum_{j=1}^{m-1} a_{j, m-j} u_{j} u_{m-j}-u_{m} \sum_{j=1}^{N} a_{m, j} u_{j}-\sigma_{m} u_{m} \\
\varepsilon \partial_{t} u_{N}=\frac{1}{2} \sum_{\substack{j+k \geq N \\
k, j<N}} a_{j, k} u_{j} u_{k},
\end{array}\right.
$$

where $x \in \Omega, y \in[0,1], t \in(0, T]$ and $2 \leq m<N$, with initial-boundary conditions

$$
\begin{cases}g_{x, 0}(y)=\left(f_{0}\right)_{x}(y), A_{x}(y, 0)=y & \text { if } x \in \Omega, 0 \leq y \leq 1 \\ u_{i}(x, 0)=u_{0 i}(x) & \text { if } x \in \Omega, 1 \leq i \leq N \\ \partial_{n} u_{i}(x, t)=0 & \text { if } x \in \partial \Omega_{0}, t \in(0, T], 1 \leq i<N \\ \partial_{n} u_{i}(x, t)=-\gamma_{i} u_{i}(x, t) & \text { if } x \in \partial \Omega_{1}, t \in(0, T], 1 \leq i<N .\end{cases}
$$

Definition 3.2. The $(N+2)$-ple $\left(A, g, u_{1}, \cdots, u_{N}\right)$ is called a solution of problem (24)-(25) in $[0, T]$ if

(i) $g \in \mathcal{L}\left(\Omega ; C\left([0, T] ; X_{[0,1]}\right)\right)$;

(ii) $A, \partial_{t} A \in L^{\infty}(\Omega ; C([0,1] \times[0, T] ;[0,1]))$;

(iii) $u_{i} \in C\left(\bar{Q}_{T}\right)$ and $u_{i} \geq 0$ in $Q_{T}$ for $1 \leq i \leq N$;

(iv) for a.e. $x \in \Omega, A_{x}$ satisfies (24) 1 and $A_{x}(y, 0)=y$ for $y \in[0,1]$;

(v) equation $(24) 2$ for $g$ is satisfied in a weak sense for a.e. $x \in \Omega$ : for all $\tau \in(0, T]$ and $\phi \in C([0,1] \times[0, T])$ with $\partial_{t} \phi \in C([0,1] \times[0, T])$

$$
\begin{aligned}
& \int \phi(y, \tau) d g_{x, \tau}(y)-\int \phi(y, 0) d\left(f_{0}\right)_{x}(y)-\int_{0}^{\tau}\left(\int \partial_{t} \phi(y, t) d g_{x, t}(y)\right) d t \\
& =\int_{0}^{\tau} \eta \chi\left[\int_{0}^{1} \phi(y, t) \partial_{y} A_{x}(y, t)\left(\int P\left(t, A_{x}(\xi, t), A_{x}(y, t)\right) d g_{x, t}(\xi)\right) d y-\int \phi(y, t) d g_{x, t}(y)\right] d t
\end{aligned}
$$

(vi) if $1 \leq i<N, u_{i} \in L^{2}\left([0, T] ; H^{1}(\Omega)\right)$ and

$$
\begin{gathered}
d_{i} \int_{0}^{T}\left[\int_{\Omega} \nabla u_{i}(x, s) \cdot \nabla \psi(x, s) d x \mid+\gamma_{i} \int_{\partial \Omega_{1}} u_{i}(x, s) \psi(x, s) d \sigma(x)\right] d s \\
=\varepsilon \iint_{Q_{T}} u_{i} \partial_{t} \psi+\varepsilon \int_{\Omega} u_{0 i} \psi(x, 0) d x+\iint_{Q_{T}} \tilde{R}_{i} \psi
\end{gathered}
$$

for all $\psi \in H^{1}\left([0, \tau] ; H^{1}(\Omega)\right), \psi(x, \tau)=0$, where $\tilde{R}_{i}$ is defined as in (24);

(vii) $\partial_{t} u_{N} \in C\left(\bar{Q}_{T}\right), u_{N}(\cdot, 0)=u_{0 N}$ in $\Omega$, and the equation for $u_{N}$ in (24) is satisfied in $Q_{T}$.

In the remainder of this section we prove the equivalence of problems (6)-(86) and (24)-(25). The following result is a first step in this direction.

Theorem 3.3. Let hypotheses $\left(H_{1-6}\right)$ be satisfied. Let $\left(A, g, u_{1}, \cdots, u_{N}\right)$ be a solution of (24) -(25) in $[0, T]$ and set, for a.e. $x \in \Omega$,

$$
f_{x, t}:=A_{x}(\cdot, t)_{\#} g_{x, t} \quad \text { for all } t \in[0, T] .
$$

Then $\left(f, u_{1}, \ldots, u_{N}\right)$ is a solution of problem (6) -(8) in $[0, T]$. 
Proof. Since, for a.e. $x \in \Omega, g_{x, t}$ is a Borel regular probability measure in $[0,1]$ for $t \in[0, T]$, so is $f_{x, t}$. By (18), for a.e. $x \in \Omega$ the function $y \rightarrow A_{x}(y, t)$ is injective for $t \in[0, T]$, so that, by [16], Theorem 1.18,

$$
\operatorname{supp} f_{x, t}=A_{x}\left(\operatorname{supp} g_{x, t}, t\right) \subseteq A_{x}([0,1], t)=\left[A_{x}(0, t), 1\right] .
$$

In particular $f_{x, t} \in X_{[0,1]}$ for a.e. $x \in \Omega$. In addition, by Remark 2.3(b) and Proposition $\mathbf{A . 1 0}$, the map $t \mapsto f_{x, t}$ belongs to $C\left([0, T] ; X_{[0,1]}\right)$ for a.e. $x \in \Omega$.

Let, for a.e. $x \in \Omega, v$ be defined by (3) and $J$ by (15). By (28) and (11), $\int P(t, b, a) d f_{x, t}(b)=0$ if $a<A_{x}(0, t)$, whence

$$
\operatorname{supp} J_{x, t} \subset\left[A_{x}(0, t), 1\right] .
$$

To avoid cumbersome notations, we set $B_{x}(\cdot, t):=A_{x}^{-1}(\cdot, t)$. Since

$$
A_{x}(\cdot, t):[0,1] \rightarrow\left[A_{x}(0, t), A_{x}(1, t)\right]=\left[A_{x}(0, t), 1\right],
$$

$B_{x}(\cdot, t)$ is well defined in $\left[A_{x}(0, t), 1\right], B_{x}\left(A_{x}(y, t), t\right) \equiv y$ for $y \in[0,1]$, and $A_{x}\left(B_{x}(a, t), t\right) \equiv a$ for $a \in\left[A_{x}(0, t), 1\right]$. Since supp $f_{x, t} \subset\left[A_{x}(0, t), 1\right]$, integrals of functions of $B(\cdot, t)$ with respect to $f_{x, t}$ are well defined.

By Definition 3.2 $(i v), \partial_{t} A_{x}(y, t)=v_{x}\left(A_{x}(y, t), t\right)$ for a.e. $x \in \Omega$. By (18), $B_{x}$ is Lipschitz continuous with respect to $y$ for a.e. $x \in \Omega$. Differentiating the identity $A_{x}\left(B_{x}(y, t), t\right)=y$ with respect to $t$ and $y$, we obtain that

$$
\left\{\begin{array}{l}
\partial_{y} A_{x}\left(B_{x}(a, t), t\right) \partial_{t} B_{x}(a, t)=-\partial_{t} A_{x}\left(B_{x}(a, t), t\right)=-v_{x}\left(A_{x}\left(B_{x}(a, t)\right), t\right)=-v_{x}(a, t) \\
\partial_{y} A_{x}\left(B_{x}(y, t), t\right) \partial_{y} B_{x}(y, t)=1
\end{array}\right.
$$

so that $\partial_{t} B_{x}(y, t) \partial_{y} A_{x}\left(B_{x}(y, t), t\right) \partial_{y} B_{x}(y, t)=\partial_{t} B_{x}(y, t)$ and, by (30),

$$
\partial_{t} B_{x}(y, t)=-v_{x}(y, t) \partial_{y} B_{x}(y, t) .
$$

Let $\psi \in C^{1}([0,1] \times[0, T])$. Let $x$ be fixed such that $A_{x}, \partial_{t} A_{x} \in C\left([0, T] ; X_{[0, t]}\right)$, and set

$$
\phi(y, t)=\psi\left(A_{x}(y, t), t\right) \quad \text { for } y \in[0,1]
$$

and

$$
C_{\phi}=-\int \phi(y, \tau) d g_{x, \tau}(y)+\int \phi(y, 0) d\left(f_{0}\right)_{x}(y)=-\int \phi\left(B_{x}(a, \tau), \tau\right) d f_{x, \tau}(a)+\int \phi(a, 0) d\left(f_{0}\right)_{x}(a) .
$$

Since $\phi$ satisfies the conditions in Definition $3.2(v)$, it follows that

(32)

$$
\begin{aligned}
& -\int_{0}^{T}\left(\int \phi_{t}(y, t) d g_{x, t}(y)\right) d t \\
& =\int_{0}^{T} \eta \chi\left[\int_{0}^{1} \phi(y, t) \partial_{y} A_{x}(y, t)\left(\int P\left(t, A_{x}(\xi, t), A_{x}(y, t)\right) d g_{x, t}(\xi)\right) d y-\int \phi(y, t) d g_{x, t}(y)\right] d t+C_{\phi} \\
& =\int_{0}^{T} \eta \chi\left[\int _ { 0 } ^ { 1 } \phi \left(B_{x}\left(A_{x}(y, t), t\right) \partial_{y} A_{x}(y, t)\left(\int P\left(t, b, A_{x}(y, t)\right) d f_{x, t}(b)\right) d y\right.\right. \\
& -\int \phi\left(B_{x}\left(A_{x}(y, t), t\right) d g_{x, t}(y)\right] d t+C_{\phi} \\
& =\int_{0}^{T} \eta \chi\left[\int_{A_{x}(0, t)}^{A_{x}(1, t)} \phi\left(B_{x}(a, t), t\right)\left(\int P(t, b, a) d f_{x, t}(b)\right) d a-\int \phi\left(B_{x}\left(A_{x}(y, t), t\right) d g_{x, t}(y)\right] d t+C_{\phi},\right.
\end{aligned}
$$

where we have used Theorem 1.19 in [16] and the relation $d a=\partial_{y} A(x, y, t) d y$.

On the other hand, the left-hand side of (32) can be written as

$$
-\int_{0}^{T}\left(\int \phi_{t}(y, t) d g_{x, t}(y)\right) d t=-\int_{0}^{T}\left(\int \phi_{t}\left(B_{x}(a, t), t\right) d f_{x, t}(a)\right) d t .
$$


Let $a \in\left[A_{x}(0, t), 1\right]$. Then $\psi(a, t)=\phi\left(B_{x}(a, t), t\right)$ and

$$
\partial_{t} \psi(a, t)=\partial_{y} \phi\left(B_{x}(a, t), t\right) \partial_{t} B_{x}(a, t)+\phi_{t}\left(B_{x}(a, t), t\right) .
$$

Since, by (28), $\operatorname{supp} f_{x, t} \subseteq\left[A_{x}(0, t), 1\right]$, it follows from (32)-(34) that

$$
\begin{aligned}
-\int_{0}^{T} & \left(\int \partial_{t} \psi(a, t) d f_{x, t}(a)\right) d t=-\int_{0}^{T}\left(\int \partial_{y} \phi\left(B_{x}(a, t), t\right) \partial_{t} B_{x}(a, t) d f_{x, t}(a)\right) d t \\
& +\int_{0}^{T} \eta \chi\left[\int_{A_{x}(0, t)}^{A_{x}(1, t)} \phi\left(B_{x}(a, t), t\right)\left(\int P(t, b, a) d f_{x, t}(b)\right) d a\right. \\
& \left.-\int \phi\left(B_{x}(a, t), t\right) d f_{x, t}(a)\right] d t+C_{\phi} \\
= & -\int_{0}^{T}\left(\int \partial_{y} \phi(B(x, a, t), t) \partial_{t} B(x, a, t) d f_{x, t}(a)\right) d t \\
& +\int_{0}^{T} \eta \chi\left[\int_{A_{x}(0, t)}^{A_{x}(1, t)} \psi(a, t)\left(\int P(t, b, a) d f_{x, t}(b)\right) d a-\int \psi(a, t) d f_{x, t}(a)\right] d t+C_{\phi} \\
= & -\int_{0}^{T}\left(\int \partial_{y} \phi\left(B_{x}(a, t), t\right) \partial_{t} B_{x}(a, t) d f_{x, t}(a)\right) d t+\int_{0}^{T}\left(\int \psi(a, t) d J_{x, t}(a)\right) d t+C_{\phi} .
\end{aligned}
$$

By (31),

$$
\begin{aligned}
\int \partial_{y} \phi\left(B_{x}(a, t), t\right) \partial_{t} B_{x}(a, t) d f_{x, t}(a) & =\int \partial_{y} \phi\left(B_{x}(a, t), t\right) \partial_{a} B_{x}(a, t) v_{x}(a, t) d f_{x, t}(a) \\
& =\int \partial_{a} \psi(a, t) v_{x}(a, t) d f_{x, t}(a)
\end{aligned}
$$

whence, by (35), the first equation in (6) is satisfied in the sense of distributions:

$$
\begin{aligned}
& -\int_{0}^{T}\left(\int \partial_{t} \psi(a, t) d f_{x, t}(a)\right) d t=\int_{0}^{T}\left(\int \partial_{a} \psi(a, t) v_{x}(a, t) d f_{x, t}(a)\right) d t \\
& \quad+\int_{0}^{T}\left(\int \psi(a, t) d J_{x, t}(a)\right) d t-\int \psi(a, \tau) d f_{x, \tau}(y)+\int \psi(a, 0) d\left(f_{0}\right)_{x}(a) .
\end{aligned}
$$

Concerning the Smoluchowski system in (6), ii is enough to observe that the third equation in (24) and the second equation in (6) coincide, since

$$
\int\left(\mu_{0}+A_{x}(\xi, t)\right)\left(1-A_{x}(\xi, t)\right) d g_{x, t}(\xi)=\int\left(\mu_{0}+a\right)(1-a) d f_{x, t}(a) .
$$

Here we have used again Theorem 1.19 in [16].

The proof that problems (6) $-(\sqrt{6})$ and (24)-(25) are equivalent is completed by the following result.

Theorem 3.4. Let $\left(f, u_{1}, \cdots, u_{N}\right)$ be a solution of (6) -(8) in $[0, T]$ and let $A_{x}(y, t)$ be defined by (16). Then there exists a probability measure $g_{x, t}$ such that

$$
f_{x, t}:=A_{x}(\cdot, t)_{\#} g_{x, t},
$$

and $\left(A, g, u_{1}, \ldots, u_{N}\right)$ is a solution of problem (24)-(25) in $[0, T]$.

Proof. As before we reason for a.e. $x \in \Omega$. Fixing such $x \in \Omega$, and also an arbitrary $t \in[0, T]$, we consider the map

$$
A_{x}(\cdot, t):[0,1] \mapsto\left[A_{x}(0, t), 1\right]
$$

By Proposition 3.1 $f_{x, t}=f_{x, t}\left\llcorner\left[A_{x}(0, t), 1\right]\right.$. Hence, by Theorem 1.20 in [16], there exists a Radon measure $g_{x, t}$ on $[0,1]$ such that

$$
f_{x, t}=f_{x, t}\left\llcorner\left[A_{x}(0, t), 1\right]=A_{x}(\cdot, t)_{\#} g_{x, t} .\right.
$$

Obviously $g_{x, t}$ is a probability measure and belongs to $X_{[0,1]}$. By Corollary A.10 the map $t \mapsto g_{x, t}$ is continuous with respect to the Wasserstein metric. In addition, $g_{x, t} \rightarrow\left(f_{0}\right)_{x}$ as $t \rightarrow 0$ since 
$A_{x}(y, 0)=y$. Therefore $g$ satisfies the qualitative assumptions in order to be a solution of (24) and (25).

To complete the proof of the theorem, it is enough to check the identities in the proof of Theorem 3.3 in the opposite direction.

\section{Local Existence And uniqueness}

By Theorems 3.3 and 3.4 problems (6)-(8) and (24)-(25) are equivalent. In this section we prove local (w.r.t. $t$ ) existence and uniqueness of a solution of problem (24)-(25). In section 5 we shall show that this solution can be continued in $[0, T]$, which completes the proof of the main result, Theorem 2.4

So in this section we have to prove:

Theorem 4.1. Let $\Omega \subset \mathbb{R}^{n}$ be an open and bounded set with a smooth boundary $\partial \Omega$, which is the disjunct union of smooth manifolds $\partial \Omega_{0}$ and $\partial \Omega_{1}$. Let $T>0$ and $N \in \mathbb{N}$, and let hypotheses $\left(H_{1-6}\right)$ be satisfied. Then there exists $\tau \in(0, T]$ such that problem (24)-(25) has a unique solution in $[0, \tau]$.

The proof is based on a contraction argument. To this purpose we introduce a suitable metric space.

Definition 4.2. Let $\tau \in(0, T]$ be given. We denote by $\left(\mathcal{X}_{\tau}, d\right)$ the complete metric space

$$
\mathcal{X}_{\tau}:=L^{\infty}(\Omega ; C([0,1] \times[0, \tau] ;[0,1])) \times \times C\left(\bar{\Omega} \times[0, \tau] ; \mathbb{R}^{N}\right),
$$

where $L^{\infty}(\Omega ; C([0,1] \times[0, \tau] ;[0,1]))$, and $C\left(\bar{\Omega} \times[0, \tau] ; \mathbb{R}^{N}\right)$ are endowed with their natural metrics as normed spaces, and $\mathcal{L}\left(\Omega ; C\left([0, T] ; X_{[0,1]}\right)\right)$ is endowed with the metric

$$
\sup _{x \in \Omega} \max _{t \in[0, T]} \mathcal{W}_{1}\left(f_{x, t}, g_{x, t}\right)
$$

(notice that condition (13) passes to the limit with respect to the $\mathcal{W}_{1}$-convergence, by Proposition A.7).

We denote by $\mathcal{X}_{\tau, \rho}$ the closed ball in $\mathcal{X}_{\tau}$ of radius $\rho>0$ centered at $\left(y, f_{0}, u_{0}\right)$.

Observe that, for the moment, we have given up the nonnegativity of $u_{i}$, which will be recovered during the proof of Theorem 4.1. For this reason we define $\mathcal{S}$ also for negative values of $u_{i}$, by requiring that $\mathcal{S}$ is even with respect to $u_{i}$ for each $i=1, \cdots, N-1$.

We must construct the map to which we can apply the contraction argument. We shall do this step by step.

Lemma 4.3. Let $(\hat{A}, g, u) \in \mathcal{X}_{T}$ and set, for a.e. $x \in \Omega$,

$$
\hat{v}_{x}(a, t):=\int \mathcal{G}_{x}\left(a, \hat{A}_{x}(\xi, t)\right) d g_{x, t}(\xi)+\mathcal{S}\left(x, a, u_{1}, \ldots, u_{N-1}\right) \geq 0 .
$$

Then, for a.e. $x \in \Omega$, the Cauchy problem

$$
\left\{\begin{array}{l}
\partial_{t} \underline{A}_{x}(y, t)=\hat{v}_{x}\left(\underline{A}_{x}(y, t), t\right) \quad \text { for } t>0 \\
\underline{A}_{x}(y, 0)=y \in[0,1]
\end{array}\right.
$$

has a unique solution defined for all $t \in(0, T]$, and the function $y \mapsto \underline{A}_{x}(y, t)$ is continuous, strictly increasing (and thus open) on $[0,1]$ and maps $[0,1]$ onto $\left[\underline{A}_{x}(0, t), 1\right]$ for all $t \in[0, T]$. Finally, the map $(x, y, t) \mapsto \underline{A}_{x}(y, t)$ belongs to $L^{\infty}(\Omega ; C([0,1] \times[0, T] ;[0,1])$.

Proof. We claim that, for a.e. $x \in \Omega$, the map $(a, t) \mapsto \hat{v}_{x}(a, t)$ is continuous and Lipschitz continuous with respect to $a \in[0,1]$, uniformly in $t \in[0, T]$.

By (10) this is trivial for the map $(a, t) \mapsto \mathcal{S}\left(x, a, u_{1}(x, t), \ldots, u_{N-1}(x, t)\right)$, since $(x, t) \mapsto$ $\left(u_{1}, \ldots, u_{N-1}\right)$ is continuous on $\bar{\Omega} \times[0, T]$ and $\left(u_{1}, \ldots, u_{N-1}\right)$ belongs to a compact set of $\mathbb{R}^{N-1}$. It remains to show that $(a, t) \mapsto \int \mathcal{G}_{x}\left(a, \hat{A}_{x}(\xi, t)\right) d g_{x, t}(\xi)$ is continuous and uniformly Lipschitz continuous with respect to $a \in[0,1]$. 
Let $a_{0}, a \in[0,1]$ and $t_{0}, t \in(0, T]$ be given. Then

$$
\begin{aligned}
& \left|\int \mathcal{G}_{x}\left(a, \hat{A}_{x}(\xi, t)\right) d g_{x, t}(\xi, t)-\int \mathcal{G}_{x}\left(a_{0}, \hat{A}_{x}\left(\xi, t_{0}\right)\right) d g_{x, t_{0}}(\xi)\right| \\
& \leq\left|\int \mathcal{G}_{x}\left(a, \hat{A}_{x}(\xi, t)\right) d g_{x, t}(\xi)-\int \mathcal{G}_{x}\left(a_{0}, \hat{A}_{x}\left(\xi, t_{0}\right)\right) d g_{x, t}(\xi, t)\right| \\
& \quad+\left|\int \mathcal{G}_{x}\left(a_{0}, \hat{A}_{x}\left(\xi, t_{0}\right)\right) d g_{x, t}(\xi)-\int \mathcal{G}_{x}\left(a_{0}, \hat{A}_{x}\left(\xi, t_{0}\right)\right) d g_{x, t_{0}}(\xi)\right|:=I_{1}+I_{2} .
\end{aligned}
$$

Since $(a, \xi, t) \mapsto \mathcal{G}_{x}\left(a, \hat{A}_{x}(\xi, t)\right)$ is uniformly continuous in $[0,1]^{2} \times[0, T], I_{1} \rightarrow 0$ as $(a, t) \rightarrow\left(a_{0}, t_{0}\right)$. Since $\xi \mapsto \mathcal{G}_{x}\left(a_{0}, \hat{A}_{x}\left(\xi, t_{0}\right)\right)$ is continuous in $\left.[0,1]\right)$ and $t \mapsto g_{x, t}$ is narrowly continuous (see Proposition A.7), also $I_{2} \rightarrow 0$ as $t \rightarrow t_{0}$.

Similarly, by (9), for a.e. $x \in \Omega$ and all $\xi \in[0,1]$ and $t \in[0, T]$,

$$
\left|\mathcal{G}_{x}\left(a, \hat{A}_{x}(\xi, t)\right)-\mathcal{G}_{x}\left(a^{\prime}, \hat{A}_{x}(\xi, t)\right)\right| \leq C\left|a-a^{\prime}\right| \quad \text { for } a, a^{\prime} \in[0,1] .
$$

This completes the proof of the claim, which implies, for a.e. $x \in \Omega$, the existence and uniqueness of the solution problem (37) for all $y \in[0,1]$. By a standard argument,

$$
\partial_{y} \underline{A}_{x}(y, t)=\exp \left[\int_{0}^{t} \partial_{a} \hat{v}_{x}\left(\underline{A}_{x}(y, s), s\right) d s\right]>0,
$$

so $0<C_{1} \leq \partial_{y} \underline{A}_{x}(y, t) \leq C_{2}$ for some constants $C_{1}$ and $C_{2}$ which depend on the compact set $K \subset \mathbb{R}^{N-1}$ which contains $\left(u_{1}(x, t), \ldots, u_{N-1}(x, t)\right)$.

Remark 4.4. It follows from the proof of Lemma 4.3 (in particular from (38)) that $\underline{A}_{x}(\xi, s)$ is Lipschitz continuous in $\xi$, uniformly with respect to $x$ and $s$.

Lemma 4.5. Let $(\hat{A}, g, u) \in \mathcal{X}_{T}$. Let, for a.e. $x \in \Omega, \underline{A}$ be defined as in Lemma 4.3 and $(F[g])_{x, t}$ be the signed measure on $[0,1]$ defined by

$$
d(F[g])_{x, t}=\eta(t) \chi(x, t)\left[\partial_{y} \underline{A}_{x}(y, t) \int P\left(t, \underline{A}_{x}(\xi, t), \underline{A}_{x}(y, t)\right) d g_{x, t}(\xi) d y-d g_{x, t}(y)\right]
$$

for $0<t \leq T$. Then, for a.e. $x \in \Omega$,

(i) the integral equation

$$
\underline{g}_{x, t}=\left(f_{0}\right)_{x}+\int_{0}^{t}(F[\underline{g}])_{x, s} d s
$$

has a unique solution $t \mapsto \underline{g}_{x, t}$ which belongs to $C\left([0, T], X_{[0,1]}\right)[1]$

(ii) the measure $\underline{g}_{x, t}$ is a weak solution of the system

$$
\left\{\begin{array}{l}
\partial_{t} \underline{g}_{x, t}(y)=\eta \chi\left[\partial_{y} \underline{A}_{x}(y, t) \int P\left(t, \underline{A}_{x}(\xi, t), \underline{A}_{x}(y, t)\right) d \underline{g}_{x, t}(\xi)-\underline{g}_{x, t}(y)\right] \\
\underline{g}_{x, 0}=\left(f_{0}\right)_{x}
\end{array}\right.
$$

in the sense of (26).

Proof. First of all, we observe that for a.e. $x \in \Omega$ and $s \in[0, T]$ and for all $g \in X_{[0,1]}$,

$$
\int d(F[g])_{x, s}=0
$$

${ }^{1}$ If $t \rightarrow \mu(t)$ is a continuous map from $[0, T]$ to $X_{[0,1]}$, for any Borel set $\mathcal{B} \subset[0,1]$, we set

$$
\left(\int_{0}^{t} \mu(s) d s\right)(\mathcal{B}):=\int_{0}^{t} \mu(s)(\mathcal{B}) d s
$$


The assertion is obvious if $\chi(x, s)=0$. If $\chi(x, s)=1$, by Tonelli's theorem,

$$
\begin{aligned}
\frac{1}{\eta} \int d(F[g])_{x, s} & \left.=\int\left(\int P\left(s, \underline{A}_{x}(\xi, s), \underline{A}_{x}(y, s)\right) \partial_{y} \underline{A}_{x}(y, s)\right) d y\right) d g_{x, s}(\xi)-\int d g_{x, s}(y) \\
& =\int\left(\int P\left(s, \underline{A}_{x}(\xi, s), b\right) d b\right) d g_{x, s}(\xi)-\int d g_{x, s}(y)=0 .
\end{aligned}
$$

We set, for a.e. $x \in \Omega$ (from now on we fix such $x$ ),

$$
q_{t}:=e^{\int_{0}^{t} \eta(s) \chi(x, s) d s} g_{x, t} \quad \text { for } t \in[0, T] .
$$

Let $Y$ be the set of such $q$, i.e. $q \in Y$ if the map $t \mapsto e^{-\int_{0}^{t} \eta(s) \chi(x, s) d s} q_{t}$ belongs to $C\left([0, T], X_{[0,1]}\right)$. Then $Y$ naturally inherits a metric from $C\left([0, T], X_{[0,1]}\right)$,

$$
d_{Y}\left(q_{1}, q_{2}\right):=\sup _{t \in(0, T)} \mathcal{W}_{1}\left(e^{-\int_{0}^{t} \eta(s) \chi(x, s) d s}\left(q_{1}\right)_{t}, e^{-\int_{0}^{t} \eta(s) \chi(x, s) d s}\left(q_{2}\right)_{t}\right),
$$

so $Y$ is a complete metric space.

The equation for $g$ translates into

$$
\partial_{t} q_{t}(y)=L q_{t}(y):=\eta \chi(x, t) \partial_{y} \underline{A}_{x}(y, t) \int P\left(t, \underline{A}_{x}(\xi, t), \underline{A}_{x}(y, t)\right) d q_{t}(\xi) \geq 0,
$$

and the corresponding integral equation is

$$
q_{t}=\left(f_{0}\right)_{x}+\int_{0}^{t} L q_{s} d s \quad \text { for } t \in[0, T] .
$$

We consider the map

$$
q \mapsto\left(f_{0}\right)_{x}+\int_{0}^{t} L q_{s} d s
$$

One easily checks that, by (40), for all $q \in Y$

$$
\int d L q_{t}=0 \quad \text { for } t \in[0, T], \quad\left(f_{0}\right)_{x}+\int_{0}^{t} L q_{s} d s \in Y .
$$

If we show that for all $q_{1}, q_{2} \in Y$

$$
J_{0}\left(q_{1}, q_{2}\right):=d_{Y}\left(\left(f_{0}\right)_{x}+\int_{0}^{t} L\left(q_{1}\right)_{s},\left(f_{0}\right)_{x}+\int_{0}^{t} L\left(q_{2}\right)_{s}\right) \leq C d_{Y}\left(q_{1}, q_{2}\right),
$$

it follows from a standard contraction argument that the map (42) has a unique fixed point in a sufficiently small interval $[0, \tau]$ and that equation (41) has a unique local solution $\underline{q}$ which can be continued in $[0, T]$.

To prove (44) we use the characterisation of the $\mathcal{W}_{1}$-distance given in Proposition A.4

$$
d_{Y}\left(q_{1}, q_{2}\right)=\sup _{t \in(0, T)}\left[e^{-\int_{0}^{t} \eta(s) \chi(x, s) d s} \sup \left\{\int \phi d\left(q_{1}-q_{2}\right)_{t} ; \phi \in \operatorname{Lip}_{1}([0,1], \mathbb{R})\right\}\right] \text {. }
$$

Hence

$$
J_{0}\left(q_{1}, q_{2}\right)=\sup _{t \in(0, T)}\left[e^{-\int_{0}^{t} \eta(s) \chi(x, s) d s} \sup \left\{I_{\phi}(t) ; \phi \in \operatorname{Lip}_{1}([0,1], \mathbb{R})\right\}\right]
$$

where

$$
I_{\phi}(t):=\int \phi d \int_{0}^{t}\left(L\left(q_{1}\right)_{s}-L\left(q_{2}\right)_{s}\right) d s
$$

and $L\left(q_{1}\right)_{s}-L\left(q_{2}\right)_{s}$ is given by

$$
\eta(s) \chi(x, s)\left(\int P\left(s, \underline{A}_{x}(\xi, s), \underline{A}_{x}(y, s)\right) \partial_{y} \underline{A}_{x}(y, s) d\left(q_{1}-q_{2}\right)_{s}(\xi)\right) d y .
$$


By Tonelli's Theorem, $I_{\phi}(t)$ is equal to

$$
\begin{aligned}
& \int \phi(y) \int_{0}^{t}\left(\eta \chi\left(\int P\left(s, \underline{A}_{x}(\xi, s), \underline{A}_{x}(y, s)\right) \partial_{y} \underline{A}_{x}(y, s) d\left(q_{1}-q_{2}\right)_{s}(\xi)\right) d y\right) d s \\
& =\int_{0}^{t} \eta \chi\left(\int_{\underline{A}_{x}(0, s)}^{1} \phi\left(B_{x}(b, s)\right)\left(\int P\left(s, \underline{A}_{x}(\xi, s), b\right) d\left(q_{1}-q_{2}\right)_{s}(\xi)\right) d b\right) d s \\
& =\int_{0}^{t} \eta \chi\left(\int\left(\int_{\underline{A}_{x}(0, s)}^{1} \phi\left(B_{x}(b, s)\right) P\left(s, \underline{A}_{x}(\xi, s), b\right) d b\right) d\left(q_{1}-q_{2}\right)_{s}(\xi)\right) d s .
\end{aligned}
$$

By (43), $I_{\phi}(t)=0$ if $\phi$ is constant, so we may assume that $\phi(0)=0$. Hence $|\phi| \leq 1$ and, by (12),

$$
\begin{aligned}
& \left|\int_{\underline{A}_{x}(0, s)}^{1} \phi\left(B_{x}(b, s)\right)\left(P\left(s, \underline{A}_{x}\left(\xi^{\prime}, s\right), b\right)-P\left(s, \underline{A}_{x}\left(\xi^{\prime \prime}, s\right), b\right)\right) d b\right| \\
& \quad \leq \int_{\underline{A}(x, 0, s)}^{1}\left|P\left(s, \underline{A}_{x}\left(\xi^{\prime}, s\right), b\right)-P\left(s, \underline{A}_{x}\left(\xi^{\prime \prime}, s\right), b\right)\right| d b \leq L\left|\xi^{\prime}-\xi^{\prime \prime}\right| .
\end{aligned}
$$

Now (44) follows from (45):

Setting

$$
J_{0}\left(q_{1}, q_{2}\right) \leq T L \max _{t \in[0, T]} \eta(t) d_{Y}\left(q_{1}, q_{2}\right)
$$

$$
\underline{g}_{x, t}=e^{-\int_{0}^{t} \eta(s) \chi(x, s) d s} \underline{q}_{t} \text { for } t \in[0, T]
$$

we have completed the proof of part $(i)$ of the lemma.

Fix an $x \in \Omega$ for which (39) and (41) (for $\underline{q}$ ) are valid. Since $P$ and $\underline{A}_{x}$ are continuous functions and the map $t \mapsto \underline{g}_{x, t}$ is continuous in the weak* topology (and so is $t \mapsto \underline{q}_{t}$ ), the map

$$
(y, t) \mapsto \int P\left(t, \underline{A}_{x}(\xi, t), \underline{A}_{x}(y, t)\right) d \underline{q}_{t}(\xi)
$$

is continuous in $[0,1] \times[0, T]$. Hence $L(\underline{q}, \cdot) \in L^{\infty}((0,1) \times(0, T))$.

We set $\tilde{q}=\underline{q}-\left(f_{0}\right)_{x}$. By (41)

$$
\tilde{q}_{t}=\int_{0}^{t} L\left(\tilde{q}_{s}+\left(f_{0}\right)_{x}\right) d s \text { for } t \in[0, T]
$$

Since, by the boundedness of $L\left(\tilde{q}_{s}+\left(f_{0}\right)_{x}\right)(y), t \mapsto \tilde{q}_{t}(y)$ is absolutely continuous in $[0, T]$ for a.e. $y \in(0,1)$, this means that

$$
\int_{0}^{1} \psi(y, \tau) \tilde{q}_{\tau}(y) d y=\iint_{(0,1) \times(0, \tau)}\left[\psi_{t}(y, t) \tilde{q}_{t}(y)+\psi(y, t) L\left(\left(\tilde{q}_{t}-\left(f_{0}\right)_{x}\right)(y)\right)\right] d y d t
$$

for all $\tau \in(0, T]$ and $\psi \in L^{\infty}([0,1] \times[0, T])$ with $\psi_{t} \in L^{\infty}([0,1] \times[0, T])$.

Finally let $\phi(y, t)$ be as in the first part of the proof (we recall that $x$ is fixed). We substitute the function $\psi(y, t)=e^{-\int_{0}^{t} \eta(s) \chi(x, s) d s} \phi(y, t)$ into (46). Since

$$
\partial_{t} \psi(y, t)=e^{-\int_{0}^{t} \eta(s) \chi(x, s) d s}\left(-\eta \chi \phi(y, t)+\partial_{t} \phi(x, y, t)\right.
$$

$\psi$ and $\partial_{t} \psi$ are continuous with respect to $y$ and, by a straightforward calculation, (46) transforms into

$$
\begin{gathered}
\int \phi(y, \tau) d \underline{g}_{x, \tau}(y)-\int \psi(y, 0) d\left(f_{0}\right)_{x}(y)=-\int_{0}^{\tau}\left[\int \partial_{t} \psi(y, t) d\left(f_{0}\right)_{x}(y)\right] d t \\
\left.+\int_{0}^{\tau}\left(\int \phi(y, t) d(F[\underline{g}])_{x, t}(y)+\int \partial_{t} \phi(y, t)\right) d \underline{g}_{x, t}(y)\right) d t
\end{gathered}
$$

for all $\tau \in(0, T]$. Since $\psi(y, 0)=\phi(y, 0)$, this implies that $\underline{g}_{x, t}(y)$ satisfies the equation of the system in the sense of (26):

$$
\left.\int \phi(y, \tau) d \underline{g}_{x, \tau}(y)-\int \phi(y, 0) d\left(f_{0}\right)_{x}(y)=\int_{0}^{\tau}\left(\int \phi(y, t) d(F[\underline{g}])_{x, t}(y)+\int \partial_{t} \phi(y, t)\right) d \underline{g}_{x, t}(y)\right) d t .
$$


Let $(\hat{A}, g, u):=\left(\hat{A}, g, u_{1} \ldots, u_{N}\right) \in \mathcal{X}_{\tau, \rho}$. By Lemma 4.3, $(\hat{A}, g, u)$ uniquely defines a function $\underline{A} \in L^{\infty}(\Omega ; C([0,1] \times[0, \tau] ;[0,1])$, and, by Lemma 4.5 , $\underline{A}$ uniquely defines a measure $\underline{g} \in$ $\mathcal{L}\left(\Omega ; C\left([0, T] ; X_{[0,1]}\right)\right)$. Let $\underline{u}:=\left(\underline{u}_{1}, \ldots, \underline{u}_{N}\right)$ be the weak solution of the problem

$$
\left\{\begin{array}{l}
\varepsilon \partial_{t} \underline{u}_{m}-d_{m} \Delta \underline{u}_{m}=F_{m}(\underline{A}, \underline{g}, u) \quad(1 \leq m<N) \\
\varepsilon \partial_{t} \underline{u}_{N}=F_{N}(\underline{A}, \underline{g}, u),
\end{array} \quad \text { in } Q_{\tau}=\Omega \times(0, \tau]\right.
$$

with initial-boundary conditions

$$
\left\{\begin{array}{ll}
\underline{u}_{i}(x, 0)=u_{0 i}(x) & \text { if } x \in \Omega \\
\partial_{n} \underline{u}_{i}(x, t)=0 & \text { if } x \in \partial \Omega_{0}, t>0 \\
\partial_{n} \underline{u}_{i}(x, t)=-\gamma_{i} \underline{u}_{i}(x, t) & \text { if } x \in \partial \Omega_{1} \times(0, \tau]
\end{array} \quad(1 \leq i \leq N) .\right.
$$

Here we have set

$$
\left\{\begin{array}{l}
F_{1}(\underline{A}, \underline{g}, u):=-\sigma_{1} u_{1}-u_{1} \sum_{j=1}^{N} a_{1, j} u_{j}+C_{\mathcal{F}} \int_{0}^{1}\left(\mu_{0}+\underline{A}_{x}(\xi, t)\right)\left(1-\underline{A}_{x}(\xi, t)\right) d \underline{g}_{x, t}(\xi) \\
F_{m}(\underline{A}, \underline{g}, u):=-\sigma_{m} u_{m}+\frac{1}{2} \sum_{j=1}^{m-1} a_{j, m-j} u_{j} u_{m-j}-u_{m} \sum_{j=1}^{N} a_{m, j} u_{j} \\
F_{N}(\underline{A}, \underline{g}, u):=\frac{1}{2} \sum_{\substack{j+k>N \\
k, j<N}} a_{j, k} u_{j} u_{k} .
\end{array}\right.
$$

Observe that $F_{i} \in L^{\infty}(\Omega \times[0, \tau])(i=1, \ldots, N)$ and its norm only depends on the compact set $K \subset$ $\mathbb{R}^{n}$ containing $\left(u_{1}, \cdots, u_{N}\right)$. We also observe that system (48)-(49) consists of $N-1$ (uncoupled) scalar linear heat equations with linear boundary conditions and an ordinary differential equation. Therefore it has a unique weak solution $\underline{u}$. More precisely, following [17] and recalling that $\mathcal{X}_{\tau, \rho}$ denotes the closed ball of radius $\rho>0$ centered at $\left(y, f_{0}, u_{0}\right)$ in $\mathcal{X}_{\tau}$, we have that

Proposition 4.6 ([17, Theorems 2.11, 3.2 and 3.3). Let $(\hat{A}, \underline{g}, u) \in X_{\tau, \rho}$. For all $1 \leq i<N$ there exists a unique $\underline{u}_{i} \in C\left([0, \tau] ; L^{2}(\Omega)\right) \cap L^{2}\left([0, \tau] ; H^{1}(\Omega)\right)$ such that

$$
\begin{gathered}
\int_{0}^{\tau}\left[\int_{\Omega} \nabla \underline{u}_{i}(x, s) \cdot \nabla \psi(x, s) d x+\gamma_{i} \int_{\partial \Omega_{1}} \underline{u}_{i}(x, s) \psi(x, s) d \sigma(x)\right] d s \\
=\iint_{Q_{\tau}} \underline{u}_{i} \partial_{t} \psi+\int_{\Omega} u_{0 i} \psi(x, 0) d x+\iint_{Q_{\tau}} F_{i}(\underline{A}, \underline{g}, u) \psi
\end{gathered}
$$

for all $\psi \in H^{1}\left([0, \tau] ; H^{1}(\Omega)\right), \psi(x, \tau)=0$. Let $\underline{u}_{N}(x, t)=u_{0 N}(x)+\int_{0}^{\tau} F_{N}(\underline{A}, \underline{g}, u) d s$ and $\underline{u}=$ $\left(\underline{u}_{1}, \cdots, \underline{u}_{N}\right)$. Then $\underline{u} \in C\left(\bar{Q}_{\tau} ; \mathbb{R}^{N}\right), \underline{u}(\cdot, 0)=\underline{u}_{0}$ and, for $1 \leq i \leq N$,

$$
\left\|\underline{u}_{i}\right\|_{C\left(\bar{Q}_{\tau} ; \mathbb{R}\right)} \leq C\left\{\left\|u_{0 i}\right\|_{L^{\infty}(\Omega)}+\left\|F_{i}\right\|_{L^{r}\left(Q_{\tau} ; \mathbb{R}\right)}\right\} \quad \text { if } r>n, \quad \frac{1}{r}+\frac{n}{2 r}<1 .
$$

In particular $\left\|\underline{u}_{i}\right\|_{C\left(\bar{Q}_{\tau} ; \mathbb{R}\right)} \leq C\left\{\left\|u_{0 i}\right\|_{C(\bar{\Omega})}+\left\|F_{i}\right\|_{C\left(\bar{Q}_{\tau} ; \mathbb{R}\right)}\right\}$.

Now we are ready to define the map to which we shall apply a contraction argument. Let $\rho>0$ be fixed. Using the notation $\underline{A}$ (Lemma 4.3), $\underline{g}$ (Lemma 4.5) and $\underline{u}$ (Proposition 4.6) introduced above, we set

$$
\mathcal{H}(\hat{A}, g, u):=(\underline{A}, \underline{g}, \underline{u}) \quad \text { for }(\hat{A}, g, u) \in \mathcal{X}_{\tau, \rho} .
$$

Let $\mathcal{T}_{d}$ denote the metric topology of $X_{\tau, \rho}$ and $\mathcal{T}$ the weaker topology on $\mathcal{X}_{\tau, \rho}$ which is obtained by endowing $L^{\infty}(\Omega ; C([0,1] \times[0, \tau] ;[0,1]))$ with the $L^{1}$-topology on $\Omega \times[0,1] \times[0, \tau]$.

Proposition 4.7. Let $\rho>0$ be fixed and let $\mathcal{H}(\hat{A}, g, u)$ be defined by (50). If $\tau>0$ is sufficiently small, then $\mathcal{H}: \mathcal{X}_{\tau, \rho} \rightarrow \mathcal{X}_{\tau, \rho},\left(\underline{A}_{n}, \underline{g}_{n}, \underline{u}_{n}\right) \rightarrow(\underline{A}, \underline{g}, \underline{u})$ in $\mathcal{T}$ if $\left(\hat{A}_{n}, g_{n}, u_{n}\right) \rightarrow(\hat{A}, g, u)$ in $\mathcal{T}_{d}$, and $\mathcal{H}$ is a contraction on $\mathcal{H}\left(X_{\tau, \rho}\right)$. 
Proof. First we prove that $\mathcal{H}\left(\mathcal{X}_{\tau, \rho}\right) \subset \mathcal{X}_{\tau, \rho}$ if $\tau$ is sufficiently small.

By Proposition 4.6. $\left.\left\|u(\cdot, t)-u_{0}\right\|_{C\left(\bar{\Omega} ; \mathbb{R}^{N}\right.}\right) \rightarrow 0$ as $t \rightarrow 0^{+}$, so it remains to show that, as $t \rightarrow 0^{+}$,

$$
\sup _{x \in \Omega, 0 \leq y \leq 1}\left|\underline{A}_{x}(y, t)-y\right| \rightarrow 0, \quad \sup _{x \in \Omega}\left\|\underline{g}_{x, t}-\left(f_{0}\right)_{x}\right\|_{X_{[0,1]}} \rightarrow 0 .
$$

Since, by (37) and assumptions (9) and (10),

$$
\begin{aligned}
\left|\underline{A}_{x}(y, t)-y\right| & \leq \int_{0}^{\tau}\left\{\int \mathcal{G}_{x}\left(\underline{A}_{x}(y, s), \hat{A}_{x}(\xi, s)\right) d g_{x, s}(\xi)+\mathcal{S}\left(x, \underline{A}_{x}(y, s), u(x, s)\right)\right\} d s \\
& \leq C_{1} \int_{0}^{\tau}\left|\underline{A}_{x}(y, s)-y\right| d s+C_{2} \tau,
\end{aligned}
$$

(51) 1 follows from Gronwall's Lemma. On the other hand, (51) 2 easily follows from Lemma $4.5(i)$ and its proof.

To prove the $\left(\mathcal{T}_{d}, \mathcal{T}\right)$-continuity of $\mathcal{H}$, let $\hat{A}_{n}, \hat{A} \in L^{\infty}(\Omega ; C([0,1] \times[0, \tau] ;[0,1])$ be such that $\left(\hat{A}_{n}, g_{n}, u_{n}\right) \rightarrow(\hat{A}, g, u)$ in $\mathcal{X}_{\tau, \rho}$ as $n \rightarrow \infty$. We must show that $A_{n} \rightarrow A$ in $L^{1}(\Omega \times[0,1] \times[0, \tau])$.

By the Dominated Convergence Theorem, this follows if

$$
\underline{A}_{n} \rightarrow \underline{A} \quad \text { a.e. in } \Omega \times[0,1] \times[0, \tau] \quad \text { as } n \rightarrow \infty .
$$

To prove (52) we observe that

$$
\begin{aligned}
&\left|\left(\underline{A}_{n}\right)_{x}(y, t)-\underline{A}_{x}(y, t)\right| \\
& \leq \int_{0}^{t}\left|\int\left[\mathcal{G}_{x}\left(\left(\underline{A}_{n}\right)_{x}(y, s),\left(\hat{A}_{n}\right)_{x}(\xi, s)\right)-\mathcal{G}_{x}\left(\underline{A}_{x}(y, s), \hat{A}_{x}(\xi, s)\right)\right] d\left(g_{n}\right)_{x, s}(\xi)\right| d s \\
& \quad+\int_{0}^{t}\left|\int \mathcal{G}_{x}\left(\underline{A}_{x}(y, s), \hat{A}_{x}(\xi, s)\right) d\left(g_{n}-g\right)_{x, s}(\xi)\right| d s \\
& \quad+\int_{0}^{t}\left|\mathcal{S}\left(x,\left(\underline{A}_{n}\right)_{x}(y, s), u_{n}(x, s)\right)-\mathcal{S}\left(x,\left(\underline{A}_{n}\right)_{x}(y, s), u(x, s)\right)\right| d s \\
& \quad+\int_{0}^{t}\left|\mathcal{S}\left(x,\left(\underline{A}_{n}\right)_{x}(y, s), u(x, s)\right)-\mathcal{S}\left(x, \underline{A}_{x}(y, s), \underline{u}(x, s)\right)\right| d s \\
&=: I_{1}+I_{2}+I_{3}+I_{4},
\end{aligned}
$$

where $I_{j}=I_{j}(x, y, t)$ for $j=1,2,3,4$. It follows easily from (10) that

$$
\begin{gathered}
I_{3} \leq C_{\rho} t \sup _{x \in \Omega, 0 \leq s \leq \tau}\left|u_{n}(x, s)-u(x, s)\right| \leq C_{\rho} t d\left(\left(\hat{A}_{n}, g_{n}, u_{n}\right),(A, g, u)\right), \\
\left.I_{4} \leq C_{\rho} t \sup _{x \in \Omega, 0 \leq y \leq 1,0 \leq s \leq \tau} \mid\left(\hat{A}_{n}\right)_{x}(y, s)\right)-\hat{A}_{x}(y, s) \mid \leq C_{\rho} t d\left(\left(\hat{A}_{n}, g_{n}, u_{n}\right),(A, g, u)\right) .
\end{gathered}
$$

By (9),

$$
\begin{aligned}
I_{1} \leq & C_{\rho} \int_{0}^{t}\left\{\int\left|\left(\underline{A}_{n}\right)_{x}(y, s)-\underline{A}_{x}(y, s)\right| d\left(g_{n}\right)_{x, s}(\xi)\right\} d s \\
& \left.\left.+C_{\rho} \int_{0}^{t}\left\{\int \mid\left(\hat{A}_{n}\right)_{x}(\xi, s)\right)-\hat{A}_{x}(\xi, s)\right) \mid d\left(g_{n}\right)_{x, s}(\xi)\right\} d s \\
= & C_{\rho} \int_{0}^{t}\left|\left(\underline{A}_{n}\right)_{x}(y, s)-\underline{A}_{x}(y, s)\right| d s \\
& \left.\left.+C_{\rho} \int_{0}^{t}\left\{\int \mid\left(\hat{A}_{n}\right)_{x}(\xi, s)\right)-\hat{A}_{x}(\xi, s)\right) \mid d\left(g_{n}\right)_{x, t}(\xi)\right\} d s \\
\leq & C_{\rho} \int_{0}^{t}\left|\left(\underline{A}_{n}\right)_{x}(y, s)-\underline{A}_{x}(y, s)\right| d s+C_{\rho} t d\left(\left(\hat{A}_{n}, g_{n}, u_{n}\right),(A, g, u)\right) .
\end{aligned}
$$

Thus, by Gronwall's inequality, Proposition A.7 and the Dominated Convergence Theorem,

$$
\left|\left(\underline{A}_{n}\right)_{x}(y, t)-\underline{A}_{x}(y, t)\right| \leq I_{2}(x, y, \tau)+C_{\rho} \tau d\left(\left(\hat{A}_{n}, g_{n}, u_{n}\right),(A, g, u)\right) \rightarrow 0
$$

as $n \rightarrow \infty$. This proves (52). 
It remains to prove that $\mathcal{H}$ is a contraction on $\mathcal{H}\left(X_{\rho, \tau}\right)$ if $\tau$ is small enough. Let $\left(\hat{A}^{1}, g^{1}, u^{1}\right)$, $\left(\hat{A}^{2}, g^{2}, u^{2}\right) \in X_{\rho, \tau}$. Repeating verbatim the arguments leading to (54), we obtain that

$$
\begin{aligned}
\left|\left[\underline{A}_{x}^{1}-\underline{A}_{x}^{2}\right](y, t)\right| \leq & \int_{0}^{t}\left|\int \mathcal{G}_{x}\left(\underline{A}_{x}^{2}(y, s), \hat{A}_{x}^{2}(\xi, s)\right) d\left(g^{1}-g^{2}\right)_{x, s}(\xi)\right| d s \\
& +C_{\rho} \tau d\left(\left(\hat{A}^{1}, g^{1}, u^{1}\right),\left(\hat{A}^{2}, g^{2}, u^{2}\right)\right) .
\end{aligned}
$$

Since $\left(\hat{A}^{2}, g^{2}, u^{2}\right) \in \mathcal{H}\left(X_{\rho, \tau}\right)$, it follows from Remark 4.4 that $\hat{A}_{x}^{2}(\xi, s)$ and, by (9),

$\mathcal{G}_{x}\left(\underline{A}_{x}^{2}(y, s), \hat{A}_{x}^{2}(\xi, s)\right)$ are Lipschitz continuous in $\xi$, uniformly with respect to $x$ and $s$. Thus, by Proposition A.4.

$$
\left|\underline{A}_{x}^{1}(y, t)-\underline{A}_{x}^{2}(y, t)\right| \leq C_{\rho} \int_{0}^{t} \mathcal{W}\left(g_{x, s}^{1}, g_{x, s}^{2}\right) d s \leq C_{\rho} \tau d\left(\left(\hat{A}^{1}, g^{1}, u^{1}\right),\left(A^{2}, g^{2}, u^{2}\right)\right) .
$$

Consider now $\mathcal{W}_{1}\left(\underline{g}_{x, t}^{1}, \underline{g}_{x, t}^{2}\right)$. In view of the definition of $\underline{g}^{1}, \underline{g}^{2}$, we may repeat verbatim the arguments in the proof of Lemma 4.5 and obtain that

$$
\begin{aligned}
\mathcal{W}_{1}\left(\underline{g}_{x, t}^{1} \underline{g}_{x, t}^{2}\right) & \leq C \max _{[0, T]} \eta t \sup _{0 \leq s \leq t} \mathcal{W}_{1}\left(g_{x, s}^{1}, g_{x, s}^{2}\right) \\
& \leq C \tau d\left(\left(\hat{A}^{1}, g^{1}, u^{1}\right),\left(\hat{A}^{2}, g^{2}, u^{2}\right)\right) .
\end{aligned}
$$

Finally, we estimate $\sup _{\Omega \times[0, \tau]}\left|\underline{u}^{1}-\underline{u}^{2}\right|$. Set $\underline{U}=\underline{u}^{1}-\underline{u}^{2}$ and $\underline{U}=\left(\underline{U}_{1}, \ldots, \underline{U}_{N}\right)$. Then $\underline{U}$ is a weak solution (in the sense of Proposition 4.6) of a system similar to (48)-(49), with $F_{j}$ replaced by $\tilde{F}_{j}:=F_{j}\left(\underline{A}^{1}, \underline{g}^{1}, u^{1}\right)-F_{j}\left(\underline{A}^{2}, \underline{g}^{2}, u^{2}\right), j=1, \ldots, N$, and $u_{0}$ by $\underline{U}(x, 0)=0$. By Proposition 4.6 ,

$$
\|\underline{U}\|_{C(\bar{\Omega} \times[0, \tau] ; \mathbb{R})} \leq C \sum_{i=1}^{N}\left\|\tilde{F}_{i}\right\|_{C(\bar{\Omega} \times[0, \tau] ; \mathbb{R})} .
$$

If $k>1, F_{k}$ is a polynomial in the components of $u$ and, since $u_{1}, u_{2}$ are uniformly bounded by $\rho$ in $\Omega \times[0, \tau]$,

$$
\left\|\tilde{F}_{k}\right\|_{C(\bar{\Omega} \times[0, \tau] ; \mathbb{R})} \leq C_{\rho} \sum_{i}\left\|u_{i}^{1}-u_{i}^{2}\right\|_{C(\bar{\Omega} \times[0, \tau] ; \mathbb{R})} \quad \text { if } k>1 .
$$

The same argument applies to the polynomial terms of $\tilde{F}_{1}$, so we are left with the estimate of

$$
I=\left\|\int_{0}^{1}\left(\mu_{0}+\underline{A}_{x}^{1}(\xi, t)\right)\left(1-\underline{A}_{x}^{1}(\xi, t)\right) d \underline{g}_{x, t}^{1}(\xi)-\int_{0}^{1}\left(\mu_{0}+\underline{A}_{x}^{2}(\xi, t)\right)\left(1-\underline{A}_{x}^{2}(\xi, t)\right) d \underline{g}_{x, t}^{2}(\xi)\right\|_{C(\bar{\Omega} \times[0, \tau] ; \mathbb{R})} .
$$

Arguing as above,

$$
\begin{aligned}
I \leq J_{1}+J_{2}:= & \int_{0}^{1}\left|\left(\mu_{0}+\underline{A}_{x}^{1}(\xi, t)\right)\left(1-\underline{A}_{x}^{1}(\xi, t)\right)-\left(\mu_{0}+\underline{A}_{x}^{2}(\xi, t)\right)\left(1-\underline{A}_{x}^{2}(\xi, t)\right)\right| d \underline{g}_{x, t}^{1}(\xi) \\
& +\left|\int_{0}^{1}\left(\mu_{0}+\underline{A}_{x}^{2}(\xi, t)\right)\left(1-\underline{A}_{x}^{2}(\xi, t)\right) d\left(\underline{g}^{1}-\underline{g}^{2}\right)(\xi)\right| .
\end{aligned}
$$

Repeating the arguments that yield the estimate (55), we have that

$$
\begin{aligned}
J_{1} & \left.\left.\leq C_{\rho} \sup _{x, \xi, t} \mid \underline{A}_{x}^{1}(\xi, t)\right)-\underline{A}_{x}^{2}(\xi, t)\right) \mid \int_{0}^{1} d \underline{g}_{x, t}^{1}(\xi) \\
& \left.\left.=C_{\rho} \sup _{x, \xi, t} \mid \underline{A}_{x}^{1}(\xi, t)\right)-\underline{A}_{x}^{2}(\xi, t)\right) \mid \leq C_{\rho} \tau d\left(\left(\hat{A}^{1}, g^{1}, u^{1}\right),\left(A^{2}, g^{2}, u^{2}\right)\right) .
\end{aligned}
$$

Concerning $J_{2}$, by Remark 4.4 the map $\xi \rightarrow\left(\mu_{0}+\underline{A}_{x}^{2}(\xi, t)\right)\left(1-\underline{A}_{x}^{2}(\xi, t)\right)$ is uniformly Lipschitz continuous. Thus, by Proposition $\mathrm{A.4}$ and (57),

$$
J_{2} \leq C_{\rho} \mathcal{W}_{1}\left(\underline{g}^{1}, \underline{g}^{2}\right) \leq C_{\rho} \tau d\left(\left(\hat{A}^{1}, g^{1}, u^{1}\right),\left(\hat{A}^{2}, g^{2}, u^{2}\right)\right) .
$$

Combining the estimates of $I, J_{1}, J_{2}$ with (59) and (58), we obtain that

$$
\left\|\underline{u}_{1}-\underline{u}_{2}\right\|_{C(\bar{\Omega} \times[0, \tau] ; \mathbb{R})} \leq C_{\rho} \tau d\left(\left(\hat{A}^{1}, g^{1}, u^{1}\right),\left(\hat{A}^{2}, g^{2}, u^{2}\right)\right) .
$$


It follows from (56), (57) and (60) that $\mathcal{H}$ is a contraction on $\mathcal{H}\left(\mathcal{X}_{\rho, \tau}\right)$ if $\tau$ is small enough.

To complete the proof of Theorem 4.1, we need a minor modification of the classical BanachCaccioppoli fixed point theorem:

Proposition 4.8 (Fixed Point Theorem). Let $(X, d)$ be a complete metric space and let $\mathcal{T}_{d}$ be the topology induced by $d$. Let $\mathcal{T}$ be a Hausdorff topology on $X$ which is weaker than $\mathcal{T}_{d}$. If $\mathcal{H}: X \rightarrow X$ is a contraction on $\mathcal{H}(X)$ which is $\left(\mathcal{T}_{d}, \mathcal{T}\right)$-continuous, then $\mathcal{H}$ has a unique fixed point.

Proof. We start carrying out the standard iteration procedure

$$
x_{n+1}=\mathcal{H}\left(x_{n}\right),
$$

starting from a point $x_{0} \in \mathcal{H}(X)$, so that $x_{n} \in \mathcal{H}(X)$ for all $n \geq 0$. As usual, by the completeness of $(X, d)$, we may assume that $x_{n} \rightarrow \bar{x} \in X$ as $n \rightarrow \infty$. When $\mathcal{H}$ is a contraction on all of $X$ (and hence in particular is Lipschitz continuous from $X$ to $X$ ) we can conclude the proof taking the limit as $n \rightarrow \infty$ in (61). In our case the argument has to be slightly adapted: on one side, $x_{n+1} \rightarrow \bar{x}$ as $n \rightarrow \infty$ with respect to the topology $\mathcal{T}$ (since it is weaker that $\mathcal{T}_{d}$ ), on the other hand $\mathcal{H}\left(x_{n}\right) \rightarrow \mathcal{H}(\bar{x})$ as $n \rightarrow \infty$ with respect to the topology $\mathcal{T}$, since $\mathcal{H}$ is $\left(\mathcal{T}_{d}, \mathcal{T}\right)$-continuous, Thus, by (61) we can conclude that $\bar{x}=\mathcal{H}(\bar{x})$ by the uniqueness of the limit in $\mathcal{T}$.

Proof of Theorem 4.1. By Proposition 4.7 and the Fixed Point Theorem, system (24)-(25) has a unique solution (in the sense of Definition 3.2) in $[0, \tau]$ for sufficiently small values of $\tau$ if we show the nonnegativity of $u_{i}$ :

$$
u_{i} \geq 0 \quad \text { in } \Omega \times[0, \tau] \quad(i=1, \ldots, N) .
$$

If $i=N$, (62) is trivially satisfied. If $1 \leq i<N$, (62) formally follows from the maximum principle. Below we make this precise if $i=1$. If $i>1$ the proof is even easier.

Since $f=C_{\mathcal{F}} \int_{0}^{1}\left(\mu_{0}+\hat{A}_{x}(\xi, t)\right)\left(1-\hat{A}_{x}(\xi, t)\right) d g_{x, t}(\xi)$ is nonnegative and belongs to $L^{\infty}\left(Q_{T}\right)$, there exists a sequence of smooth nonnegative functions $\left(f_{k}\right)_{k \in \mathbb{N}}$ converging to $f$ in $L^{r}\left(Q_{T}\right)$, where $r>n$ and $\frac{1}{r}+\frac{n}{2 r}<1$. We also approximate $h=\sum_{j=1}^{N} a_{1, j} u_{j} \in C\left(\bar{Q}_{T}\right)$ uniformly by smooth functions $h_{k}$. Let $v_{k}$ be the unique smooth solution of

$$
\begin{cases}\varepsilon \partial_{t} v_{k}=d_{1} \Delta v_{k}-v_{k} h_{k}+f_{k} & \text { in } Q_{\tau} \\ v_{k}(x, 0)=u_{01}(x) & \text { if } x \in \Omega \\ \partial_{n} v_{k}(x, t)=0 & \text { if } x \in \partial \Omega_{0}, t>0 \\ \partial_{n} v_{k}(x, t)=-\gamma_{i} v_{k}(x, t) & \text { if } x \in \partial \Omega_{1}, t>0 .\end{cases}
$$

Since $\gamma_{1}>0, f_{k} \geq 0$ in $Q_{\tau}$ and $u_{01} \geq 0$ in $\Omega$, it follows from the maximum principle that $v_{k} \geq$ in $Q_{\tau}$.

On the other hand $w_{k}:=u_{1}-v_{k}$ is a weak solution of

$$
\begin{cases}\varepsilon \partial_{t} w_{k}=d_{1} \Delta w_{k}-w_{k} h_{k}+f-f_{k} & \text { in } Q_{\tau} \\ w_{k}(x, 0)=0 & \text { if } x \in \Omega \\ \partial_{n} w_{k}(x, t)=0 & \text { if } x \in \partial \Omega_{0}, t>0 \\ \partial_{n} w_{k}(x, t)=-\gamma_{i} w_{k}(x, t) & \text { if } x \in \partial \Omega_{1}, t>0,\end{cases}
$$

and it follows from [17, Theorem 3.2, that $v_{k} \rightarrow u_{1}$ uniformly on $\bar{Q}_{\tau}$. Therefore also $u_{1} \geq 0$ in $Q_{\tau}$.

\section{Global existence}

In this section we complete the proof of Theorem 2.4 by showing that the local solution of problem (24)-(25), constructed in the previous section, can be continued to the whole interval $[0, T]$. We recall that problems (24)-(25) and (6) $-(\underline{60})$ are equivalent, as we have shown in section 3.

Arguing by contradiction we suppose that the maximal interval of existence is $\left[0, \tau^{*}\right)$ for some $\tau^{*}<T$. 
A priori estimate for $u(x, t)$. Since

$$
C_{\mathcal{F}} \int_{0}^{1}\left(\mu_{0}+A_{x}(\xi, t)\right)\left(1-\hat{A}_{x}(\xi, t)\right) d g_{x, t}(\xi) \leq C_{1} \quad \text { in } \Omega \times\left[0, \tau^{*}\right)
$$

for some constant $C_{1}$, it follows formally from the maximum principle that

$$
u_{1}(x, t) \leq \sup _{\Omega} u_{01}+C_{1} t \quad \text { for } x \in \Omega, 0 \leq t<\tau^{*} .
$$

Similarly, if $u_{1}, \cdots, u_{m-1}$ are bounded in $L^{\infty}\left(\Omega \times\left[0, \tau^{*}\right)\right)$ for some $1<m<N$, then

$$
\frac{1}{2} \sum_{j=1}^{m-1} a_{j, m-j} u_{j} u_{m-j} \leq C_{m} \quad \text { in } \Omega \times\left[0, \tau^{*}\right)
$$

for some constant $C_{m}$, and it follows formally from the maximum principle that

$$
u_{m}(x, t) \leq \sup _{\Omega} u_{0 m}+C_{m} t \quad \text { for } x \in \Omega, 0 \leq t<\tau^{*} .
$$

In both cases the use of the maximum principle is justified as in the proof of (62).

The boundedness of $u_{N}$ in $\Omega \times\left[0, \tau^{*}\right)$ follows from that of $u_{1}, \cdots, u_{N-1}$, so we have shown that, for some $C_{u}>0$,

$$
|u| \leq C_{u} \quad \text { in } \Omega \times\left[0, \tau^{*}\right) .
$$

Existence of $\lim _{t \rightarrow \tau^{*}} A_{x}(y, t)=: A_{x}\left(y, \tau^{*}\right)$. Arguing as in the proof of Lemma 4.3 we obtain that $A_{x}(y, t)$ and $v_{x}\left(A_{x}(y, t), t\right)$ are Lipschitz continuous with respect to $y$, uniformly with respect to $x \in \Omega$ and $t \in\left[0, \tau^{*}\right)$. By the boundedness of $v_{x}\left(A_{x}(y, t), t\right)$, the map $t \mapsto A_{x}(y, t)$ is Lipschitz continuous on $\left[0, \tau^{*}\right)$. Hence $A_{x}\left(y, \tau^{*}\right):=\lim _{t \rightarrow \tau^{*}} A_{x}(y, t)$ exists and is Lipschitz continuous with respect to $y$, uniformly with respect to $x \in \Omega$.

Existence of $\lim _{t \rightarrow \tau^{*}} g_{x, t}=: g_{x, \tau^{*}}$. We repeat verbatim the arguments of the proof of Lemma 4.5 and we obtain that the map $t \mapsto g_{x, t}$ is Lipschitz continuous from $\left[0, \tau^{*}\right)$ to $X_{[0,1]}$ endowed with Wasserstein metric $\mathcal{W}_{1}$.

Existence of $\lim _{t \rightarrow \tau^{*}} u(x, t)=: u\left(x, \tau^{*}\right)$. In view of (65) and (66), it follows from standard regularity theory for weak solutions of parabolic equations (see e.g. [20, Theorem 1, page 111]) that $u$ is uniformly (Hölder) continuous in $\Omega \times\left[0, \tau^{*}\right)$. Hence $u$ can be extended to $\Omega \times\left[0, \tau^{*}\right]$ as a continuous function.

Hence we can apply the local existence theorem to the "initial" functions $g_{x, \tau^{*}}$ and $u\left(x, \tau^{*}\right)$, and obtain a solution in $\left[\tau^{*}, \tau_{1}\right]$ for some $\tau_{1} \in\left[\tau^{*}, T\right]$. Therefore $\left[0, \tau^{*}\right)$ is not the maximal interval of existence and we have found a contradiction.

\section{ACKNOWLEDGMENTS}

The authors would like to express their gratitude to MD Norina Marcello for many stimulating and fruitful discussions over several years.

B. F. and M. C. T. are supported by the University of Bologna, funds for selected research topics, and by MAnET Marie Curie Initial Training Network. B. F. is supported by GNAMPA of INdAM (Istituto Nazionale di Alta Matematica "F. Severi"), Italy, and by PRIN of the MIUR, Italy.

A. T. is member of GNFM (Gruppo Nazionale per la Fisica Matematica) of INdAM (Istituto Nazionale di Alta Matematica "F. Severi"), Italy.

\section{REFERENCES}

1. Y. Achdou, B. Franchi, N. Marcello, and M. C. Tesi, A qualitative model for aggregation and diffusion of $\beta$-amyloid in Alzheimer's disease, J. Math. Biol. 67 (2013), no. 6-7, 1369-1392.

2. L. Ambrosio, N. Gigli, and G. Savaré, Gradient flows in metric spaces and in the space of probability measures, second ed., Lectures in Mathematics ETH Zürich, Birkhäuser Verlag, Basel, 2008.

3. M. Bertsch, B. Franchi, N. Marcello, M. C. Tesi, and A. Tosin, Alzheimer's disease: a mathematical model for onset and progression, Math. Med. Biol. 34 (2017), no. 2, 193-214. 
4. M. Bertsch, B. Franchi, M. C. Tesi, and A. Tosin, Microscopic and macroscopic models for the onset and progression of Alzheimer's disease, J. Phys. A (2017), 414003.

5. H. Braak and K. Del Tredici, Alzheimer's pathogenesis: is there neuron-to-neuron propagation?, Acta Neuropathol. 121 (2011), no. 5, 589-595.

6. J. A. Carrillo, R. M. Colombo, P. Gwiazda, and A. Ulikowska, Structured populations, cell growth and measure valued balance laws, J. Differential Equations 252 (2012), no. 4, 3245-3277.

7. J. H. M. Evers, S. C. Hille, and A. Muntean, Measure-valued mass evolution problems with flux boundary conditions and solution-dependent velocities, SIAM J. Math. Anal. 48 (2016), no. 3, $1929-1953$.

8. B. Franchi and S. Lorenzani, From a microscopic to a macroscopic model for Alzheimer disease: two-scale homogenization of the Smoluchowski equation in perforated domains, J. Nonlinear Sci. 26 (2016), no. 3, 717753.

9. $ـ$ Smoluchowski equation with variable coefficients in perforated domains: homogenization and applications to mathematical models in medicine, Harmonic analysis, partial differential equations and applications, Appl. Numer. Harmon. Anal., Birkhäuser/Springer, Cham, 2017, pp. 49-67.

10. B. Franchi and M. C. Tesi, A qualitative model for aggregation-fragmenattion and diffusion of $\beta$-amyloid in Alzheimer's disease, Rend. Semin. Mat. Univ. Politec. Torino 7 (2012), 75-84, Proceedings of the meeting "Forty years of Analysis in Torino, A conference in honor of Angelo Negro".

11. C. Haass and D. J. Selkoe, Soluble protein oligomers in neurodegeneration: lessons from the Alzheimer's amyloid beta-peptide, Nat. Rev. Mol. Cell. Biol. 8 (2007), no. 2, 101-112.

12. W. Hao and A. Friedman, Mathematical model on Alzheimer's disease, BMC Systems Biology 108 (2016), no. $10,1-18$.

13. M. Helal, E. Hingant, L. Pujo-Menjouet, and G. F. Webb, Alzheimer's disease: analysis of a mathematical model incorporating the role of prions, J. Math. Biol. 69 (2013), no. 5, 1-29.

14. J. J. Iliff, M. Wang, Y. Liao, B. A. Plogg, W. Peng, G. A. Gundersen, H. Benveniste, G. E. Vates, R. Deane, S. A. Goldman, E. A. Nagelhus, and M. Nedergaard, A paravascular pathway facilitates CSF flow through the brain parenchyma and the clearance of interstitial solutes, including amyloid $\beta$, Sci. Transl. Med. 4 (2012), no. 147, 147ra111.

15. E. Karran, M. Mercken, and B. De Strooper, The amyloid cascade hypothesis for Alzheimer's disease: an appraisal for the development of therapeutics, Nat Rev. Drug Discov. 10 (2011), no. 9, 698-712.

16. P. Mattila, Geometry of sets and measures in Euclidean spaces, Cambridge Studies in Advanced Mathematics, vol. 44, Cambridge University Press, Cambridge, 1995, Fractals and rectifiability.

17. R. Nittka, Inhomogeneous parabolic Neumann problems, Czechoslovak Math. J. 64(139) (2014), no. 3, $703-742$.

18. B. Perthame, Transport equations in biology, Frontiers in Mathematics, Birkhäuser Verlag, Basel, 2007.

19. C. Reitz, C. Brayne, and R. Mayeux, Epidemiology of Alzheimer disease, Nat. Rev. Neurol. 7 (2011), $137-152$.

20. F. Rothe, Global solutions of reaction-diffusion systems, Lecture Notes in Mathematics, vol. 1072, SpringerVerlag, Berlin, 1984

21. D. J. Selkoe and J. Hardy, The amyloid hypothesis of alzheimer's disease at 25 years, EMBO Molecular Medicine 8 (2016), no. 6, 595-608.

22. J.-M. Serot, J. Zmudka, and P. Jouanny, A possible role for CSF turnover and choroid plexus in the pathogenesis of late onset Alzheimer's disease, J. Alzheimer's Dis. 30 (2012), no. 1, 17-26.

23. O. G. Tatarnikova, M. A. Orlov, and Bobkova N.V, Beta-amyloid and tau protein: Structure, interaction and prion-like properties, Biochemistry (Moscow) 80 (2015), no. 13, 1800-1819.

\section{Appendix A. Probability measures and Wasserstein metrics}

Throughout this appendix, $X$ denotes a complete separable metric space, with metric $d$. A positive Borel measure $\mu$ on $X$ such that $\mu(X)=1$ is said a probability measure, and we write $\mu \in \mathcal{P}(X)$. Every $\mu \in \mathcal{P}(X)$ is a Radon measure (see [2]).

Definition A.1 (Push forward of measures). Let $\mathcal{B}(X)$ be the Borel $\sigma$-algebra of subsets of $X$ and $\phi: X \rightarrow X$ a Borel measurable function, i.e. one such that $\phi^{-1}(E) \in \mathcal{B}(X)$ for every $E \in \mathcal{B}(X)$. Let moreover $\mu \in \mathcal{P}(X)$. The push forward of $\mu$ through $\phi$ is the measure $\nu \in \mathcal{P}(X)$, denoted by $\nu=\phi_{\#} \mu$, such that

$$
\nu(E):=\mu\left(\phi^{-1}(E)\right), \quad \forall E \in \mathcal{B}(X) .
$$

Equivalently, the measure $\nu$ can be characterised by

$$
\int_{X} f(x) d \nu(x)=\int_{X} f(\phi(x)) d \mu(x)
$$

for every bounded Borel function $f$ defined on $X$.

Definition A.2 (Wasserstein distances). Let $p \geq 1$ and $\mu, \nu \in \mathcal{P}(X)$ be such that

$$
\int_{X} d(x, \bar{x})^{p} d \mu(x)<+\infty, \quad \int_{X} d(x, \bar{x})^{p} d \nu(x)<+\infty
$$


for some $\bar{x} \in X$. The $p$-th Wasserstein distance between $\mu$ and $\nu$ is the number denoted by $\mathcal{W}_{p}(\mu, \nu)$ and defined by

$$
\mathcal{W}_{p}^{p}(\mu, \nu):=\inf \left\{\iint_{X^{2}} d(x, y)^{p} d \gamma(x, y): \gamma \in \Gamma(\mu, \nu)\right\},
$$

where $\Gamma(\mu, \nu) \subset \mathcal{P}\left(X^{2}\right)$ is the set of all transference plans between $\mu$ and $\nu$, i.e. the set of measures $\gamma \in \mathcal{P}\left(X^{2}\right)$ whose marginals are $\mu, \nu$, respectively.

Proposition A.3 (2], Proposition 7.1.5). If $\mu \in \mathcal{P}(X)$ has compact support then for any $\bar{x} \in X$ and $p \geq 1$

$$
\int_{X} d(x, \bar{x})^{p} d \mu(x)<+\infty .
$$

In particular, $\mu$ has finite $p$-moment. We shall write $\mu \in \mathcal{P}_{p}(X)$. Endowed with the Wasserstein p-distance $\mathcal{W}_{p}, \mathcal{P}_{p}(X)$ is a complete metric space.

Proposition A.4 (Kantorovich-Rubinstein duality, cf. [2], Eq. (7.1.2)). If $\mu, \nu \in \mathcal{P}_{1}(X)$ have compact support, then

$$
\mathcal{W}_{1}(\mu, \nu)=\sup \left\{\int_{X} \phi d(\mu-\nu): \phi \in \operatorname{Lip}_{1}(X, \mathbb{R})\right\},
$$

where $\operatorname{Lip}_{1}(X, \mathbb{R})$ is the space of Lipschitz continuous functions $\phi: X \rightarrow \mathbb{R}$ with Lipschitz constant not greater than 1 .

Definition A.5. Let $\left(\mu_{n}\right)_{n \in \mathbb{N}}$ be a sequence in $\mathcal{P}(X)$. We say that

(i) $\mu_{n} \rightarrow \mu$ narrowly if for any bounded continuous function $f$

$$
\int_{X} f d \mu_{n} \rightarrow \int_{X} f d \mu \quad \text { as } n \rightarrow \infty
$$

(ii) $\mu_{n} \rightarrow \mu$ weakly* if for any compactly supported continuous function $f$

$$
\int_{X} f d \mu_{n} \rightarrow \int_{X} f d \mu \quad \text { as } n \rightarrow \infty .
$$

Remark A.6. Obviously, narrow convergence implies weak* ${ }^{*}$ convergence, and narrow and weak* convergence are equivalent if $X$ is compact.

Proposition A.7. Let $X$ be a separable metric space. Let $\left(\mu_{n}\right)_{n \in \mathbb{N}}$ be a sequence in $\mathcal{P}_{p}(X)$. We have:

(i) if $\mathcal{W}_{p}\left(\mu_{n}, \mu\right) \rightarrow 0$ as $n \rightarrow \infty$, then $\mu_{n} \rightarrow \mu$ as $n \rightarrow \infty$ weakly ${ }^{*}$;

(ii) suppose there exist a compact set $K$ such that $\operatorname{supp} \mu_{n} \subset K$ for all $n \in \mathbb{N}$ and an open set $\mathcal{O}$ satisfying

Then

$$
K \subset \mathcal{O} \quad \text { and } \quad X \backslash \mathcal{O} \neq \emptyset .
$$

$$
\mathcal{W}_{p}\left(\mu_{n}, \mu\right) \rightarrow 0 \quad \text { as } n \rightarrow \infty
$$

if and only if $\mu_{n} \rightarrow \mu$ as $n \rightarrow \infty$ weakly* (or, equivalently, narrowly).

Proof. We apply [2, Proposition 7.1.5]. We have but to prove that the $\mu_{n}$ 's have uniformly integrable $p$-moments. By [2, Lemma 5.1.7] the assertion will follow by showing that

$$
\lim _{n \rightarrow \infty} \int_{X} f(x) d \mu_{n}(x)=\int_{X} f(x) d \mu(x)
$$

for any continuous real function $f$ such that

$$
|f(x)| \leq A+B d(x, \bar{x})^{p} \quad A, B>0, \bar{x} \in X \text { fixed. }
$$

Take now a continuous map $f: X \rightarrow \mathbb{R}$ satisfying (67). By Urysohn's lemma we can easily construct a continuous function $\tilde{f}$ such that

$$
\operatorname{supp} \tilde{f} \subset \mathcal{O} \quad \text { and } \quad \tilde{f} \equiv f \text { in } K .
$$


Thus

$$
\begin{aligned}
\lim _{n \rightarrow \infty} \int_{X} f(x) d \mu_{n}(x)=\lim _{n \rightarrow \infty} \int_{K} f(x) d \mu_{n}(x)=\lim _{n \rightarrow \infty} \int_{K} \tilde{f}(x) d \mu_{n}(x) \\
\quad=\lim _{n \rightarrow \infty} \int_{X} \tilde{f}(x) d \mu_{n}(x)=\int_{X} \tilde{f}(x) d \mu(x)=\int_{K} \tilde{f}(x) d \mu(x) \\
=\int_{K} f(x) d \mu(x)=\int_{X} f(x) d \mu(x) .
\end{aligned}
$$

Remark A.8. If $X$ is compact, then the assertion is trivial. Indeed, we have already pointed out that narrow convergence and weak ${ }^{*}$ convergence are equivalent on compact metric spaces. Thus we can apply [2, Proposition 7.1.5]. Indeed the $\mu_{n}$ 's have uniformly integrable $p$-moments, by [2, Lemma 5.1.7].

Proposition A.9. Let $X, Y$ be complete separable metric spaces. In addition, let $X$ be compact and assume that for any compact set $K \subset Y$ there exists an open set $\mathcal{O}$ such that

$$
K \subset \mathcal{O} \quad \text { and } \quad Y \backslash \mathcal{O} \neq \emptyset \text {. }
$$

Let $\left(\mu_{n}\right)_{n \in \mathbb{N}}$ be a sequence in $\mathcal{P}_{p}(X)$. Let $\Phi_{n}: X \rightarrow Y$ be a sequence of continuous injective (and hence open) maps that converges uniformly to a continuous injective (and hence open) map $\Phi: X \rightarrow Y$. Then, if $p>0$

$$
\lim _{n \rightarrow \infty} \mathcal{W}_{p}\left(\mu_{n}, \mu\right)=0 \quad \text { if and only if } \quad \lim _{n \rightarrow \infty} \mathcal{W}_{p}\left(\left(\Phi_{n}\right)_{\#} \mu_{n}, \Phi_{\#} \mu\right)=0
$$

Proof. By Proposition A.7 and [2, Remark 5.1.5], the sequence $\left(\mu_{n}\right)_{n \in \mathbb{N}}$ is tight. By [2, Lemma $5.2 .1]$

$$
\lim _{n \rightarrow \infty} \mathcal{W}_{p}\left(\mu_{n}, \mu\right)=0 \quad \Rightarrow \quad\left(\Phi_{n}\right)_{\#} \mu_{n} \rightarrow \Phi_{\#} \mu
$$

narrowly as $n \rightarrow \infty$. Set set

$$
K_{0}:=\overline{\{y ; d(y, \Phi(K))<\varepsilon\}}
$$

If $n>\bar{n}$, then $\operatorname{supp}\left(\Phi_{n}\right)_{\#} \mu_{n} \subset \Phi_{n}(K) \subset K_{0}$ that is compact. By assumption, there is an open set $\mathcal{O}_{0}$ such that

$$
K_{0} \subset \mathcal{O}_{0} \quad \text { and } \quad Y \backslash \mathcal{O}_{0} \neq \emptyset \text {. }
$$

Thus, by Proposition A.7 $\lim _{n \rightarrow \infty} \mathcal{W}_{p}\left(\left(\Phi_{n}\right)_{\#} \mu_{n}, \Phi_{\#} \mu\right)=0$. This proves the first part of the statement.

Suppose now $\lim _{n \rightarrow \infty} \mathcal{W}_{p}\left(\left(\Phi_{n}\right)_{\#} \mu_{n}, \Phi_{\#} \mu\right)=0$

We notice now that the sequence $\left(\mu_{n}\right)_{n \in \mathbb{N}}$ in $\mathcal{P}(X)$ is tight (again by Remark A.6), and hence, by [2, Theorem 5.1.3], is relatively compact with respect to the narrow convergence. Therefore, there exists a subsequence $\left(\mu_{n_{j}}\right)_{j \in \mathbb{N}}$ converging narrowly to $\nu \in \mathcal{P}(X)$. By Proposition A.7 $\lim _{j \rightarrow \infty} \mathcal{W}_{p}\left(\mu_{n_{j}}, \nu\right)=0$, and then $\lim _{n \rightarrow \infty} \mathcal{W}_{p}\left(\left(\Phi_{n_{j}}\right)_{\#} \mu_{n}, \Phi_{\#} \nu\right)=0$ (by the first part of the present proposition). Thus the uniqueness of the Wasserstein limit yields $\Phi_{\#} \nu=\Phi_{\#} \mu$ and eventually $\nu=\mu$, i.e. $\lim _{j \rightarrow \infty} \mathcal{W}_{p}\left(\mu_{n_{j}}, \mu\right)=0$. A standard argument in metric spaces makes possible to recover the limit for the full sequence $\left(\mu_{n}\right)_{n \in \mathbb{N}}$.

Corollary A.10. Let $X, Y$ be complete separable metric spaces satisfying the assumption of Proposition A.9. If $I \subset \mathbb{R}$ is an interval, let $\Phi: X \times I \rightarrow Y$ be a continuous map such that for any $t \in I$ the map $x \rightarrow \Phi(x, t)$ is injective and open.

If $t \in I$, let $\mu(t) \in \mathcal{P}(X)$ such that $\operatorname{supp} \mu(t) \subset K$ for all $t \in I$, where $K \subset X$ is a compact as in Proposition A.7.

Then $t \mapsto \mu(t)$ is continuous (with respect to the Wasserstein topology) if and only if $t \mapsto$ $\Phi(\cdot, t)_{\#} \mu(t)$ is continuous (with respect to the Wasserstein topology). 
Dipartimento di Matematica, Università di Roma "Tor Vergata”, Via della Ricerca Scientifica 1, 00133 Roma, Italy

istituto per le Applicazoni del Calcolo "M. Picone", Consiglio Nazionale delle Ricerche, Via dei TAurini 19, 00185 Roma, Italy

E-mail address: bertsch@mat.uniroma2.it ITALY

University of Bologna, Department of Mathematics, Piazza di Porta S. Donato 5, 40126 Bologna, E-mar

E-mail address: bruno.franchi@unibo.it

University of Bologna, Department of Mathematics, Piazza di Porta S. Donato 5, 40126 Bologna, ITALY

E-mail address: mariacarla.tesi@unibo.it

Department of Mathematical Sciences "G. L. Lagrange", Politecnico di Torino, Corso Duca degli Abruzzi 24, 10129 ToRino, Italy

E-mail address: andrea.tosin@polito.it 